\title{
Two-Parameter Dynamics of An Autonomous Mechanical Governor System With Time Delay
}

\author{
Shuning Deng \\ Hunan University \\ Jinchen Ji \\ University of Technology Sydney \\ Guilin Wen ( $\sim$ glwen@ysu.edu.cn ) \\ Yanshan University \\ Huidong $\mathbf{X u}$ \\ Taiyuan University of Technology
}

\section{Research Article}

Keywords: Autonomous mechanical governor system, Time-delay, Two-parameter dynamics, Bifurcations analyses, Multistability phenomena, Chaotic transition

Posted Date: June 30th, 2021

DOI: https://doi.org/10.21203/rs.3.rs-564675/v1

License: (c) (1) This work is licensed under a Creative Commons Attribution 4.0 International License. Read Full License

Version of Record: A version of this preprint was published at Nonlinear Dynamics on November 15th, 2021. See the published version at https://doi.org/10.1007/s11071-021-07039-1. 


\title{
Two-parameter dynamics of an autonomous mechanical governor system with time delay
}

\author{
Shuning Deng ${ }^{1}$, Jinchen $\mathrm{Ji}^{2}$, Guilin Wen ${ }^{1,3,}$, Huidong $\mathrm{Xu}^{4}$ \\ ${ }^{1}$ State Key Laboratory of Advanced Design and Manufacture for Vehicle Body, Hunan University, \\ Changsha, Hunan 410082, China \\ ${ }^{2}$ School of Mechanical and Mechatronic Engineering, University of Technology Sydney, Ultimo, \\ NSW 2007, Australia \\ ${ }^{3}$ School of Mechanical Engineering, Yanshan University, Qinhuangdao, Hebei 066004, China \\ ${ }^{4}$ College of Mechanical and Vehicle Engineering, Taiyuan University of Technology, Taiyuan, \\ Shanxi 030024, China \\ *Corresponding author (GL Wen), E-mail: glwen@ysu.edu.cn
}

Abstract: Understanding of dynamical behavior in the parameter-state space plays a vital role in the optimal design and motion control of mechanical governor systems. By combining the GPU parallel computing technique with two determinate indicators, namely, the Lyapunov exponents and Poincaré section, this paper presents a detailed study on the two-parameter dynamics of a mechanical governor system with different time delays. By identifying different system responses in two-parameter plane, it is shown that the complexity of evolutionary process can increase significantly with the increase of time delay. The path-following strategy and the time domain collocation method are used to explore the details of the evolutionary process. An interesting phenomenon is found in the dynamical behavior of the delayed governor system, which can cause the inconsistency between the number of intersection points of certain periodic response on Poincaré section and the actual period characteristic. For example, the commonly exhibited period-1 orbit may have two or more intersection points on the Poincaré section instead of one point. Furthermore, the variations of basins of attraction are also discussed in the plane of initial history conditions to demonstrate the observed multistability phenomena and chaotic transitions.

Key Words: Autonomous mechanical governor system; Time-delay; Two-parameter dynamics; Bifurcations analyses; Multistability phenomena; Chaotic transition. 


\section{Introduction}

Time-delay effect is widely encountered in many dynamical systems including biological systems [1-3], social and economic systems [4,5], neural networks [6,7], chemical reactions [8,9], and mechanical systems [10-11], due to the essential dependency of present states on past states. Compared to the dynamical systems without time-delay effect, systems involving inherent time delays can exhibit more rich and complicated dynamics, including the instability of equilibrium, multiple stability switches induced by time-delay [12,13], high co-dimension bifurcations [14-16], coexistence of attractors $[17,18]$, and chaotic motions $[19,20]$. In addition to the understanding of delay-induced bifurcations and transitions, the beneficial applications of time-delay effect have also attracted great interest from the research community. For example, time-delay effect has been considered in the design of real-world controllers, due to the unavoidable time consumption in the measurement, signal processing, computing and transmission processes. The inherent time delays could be used to achieve the desired control objectives, such as for the traffic arrangement [21,22], signal enhancement [23], broadband energy harvesting [24,25], and absorber and isolator design [26,27].

The mechanical centrifugal governor has played a role of milestone in the field of automatic feedback control, since it was invented and used for stabilizing the working speed of steam engines. Owing to the simple yet effective characteristics, the centrifugal governor devices have been widely used in many rotating machines to avoid potential damage, such as in the internal combustion engines, fueled turbines, modern striking clocks and so on. Induced by the inherent nonlinearities, many dynamical phenomena have been observed and reported in such systems, including periodic oscillations [28,29], chaotic motions [30,31], hyperchaotic behavior [32], 
multistability [15,33], fractal and mode-locking structures [34,35]. To avoid the damages caused by the undesired system response, various control schemes have been proposed to stabilize the system. For example, the feedback controller [36,37], adaptive controller [37], and time-delayed controller [38] were used to convert the chaotic motions of the governor systems into specified motions. A synchronization-based adaptive sliding mode controller [39] and a robust adaptive controller [40] were designed to realize the finite-time stabilization of mechanical governor systems with uncertainties. The design of anti-bifurcation controllers $[41,42]$ were studied in a centrifugal governor system to generate desirable oscillations which can be applied in the fault diagnosis, monitoring, mixing and low-energy navigation.

Although remarkable achievements have been made to the understanding of dynamical behavior in the literature, few research works have been devoted to the dynamics of such governor systems under the inherent effect of time-delay. Actually, time-delay can be originated from the gear transmission, spring deformation [37], steam transportation or chemical combustion [15]. The reaction delay caused by the mass-spring structure was considered in the governor system, and varieties of dynamical responses were illustrated from numerical simulations [37]. In Ref. [15], the time-delay effect in the governor system was modeled by considering the reaction delay induced by the working principle of engines. It was found that the time delay can shrink the stability region of equilibrium and even induce high co-dimension bifurcations, e.g., resonant and non-resonant double Hopf bifurcations. However, the dynamics and transitions are not fully understood and yet to be studied in the parameter region, where the chosen equilibrium loses its stability. To develop an in-depth insight into the open questions unaddressed in Ref. [15], this paper aims to reveal the global dynamics of the autonomous governor system with and without time 
delay, e.g., the two-parameter classification of system response and the related multistability phenomena, which will develop a deep understanding of time-delay induced global dynamical behavior of the present governor system.

Indeed, the general studies on the nonlinear dynamics were mainly focused on the local bifurcation analysis during past decades, which only provides a limited view on the system performance. With the development of computer technology, it has been possible for the researchers to study a wide range of bifurcation behaviors of certain systems in the parameter and state spaces. It is worth mentioning that an efficient accelerated tool, i.e., the CUDA-based GPU parallel computing technique [43], has been rapidly developed for the computation of multi-parameter dynamics of the systems and the efficiency of such a numerical tool has been proved in many works [44-46]. However, these works are basically directed to the dynamical systems described by the ordinary differential equations, few research works are associated with the global dynamics computation for the dynamical systems governed by the delayed differential equations (i.e., functional differential equations). In this paper, the CUDA-based GPU parallel computing technique is adopted to study the classification of two-parameter dynamics in a time-delayed governor system. Therefore, this work can be regarded as an important attempt in the computation of global dynamics of the delayed dynamical system.

Given that the existing numerical methods are basically for the ordinary differential dynamical systems, the treatment of the delayed terms and transforming the delayed differential dynamical system into the ordinary differential dynamical system then become a noteworthy concern in the computation of global dynamics of the delayed systems. Currently, the efficient approximation schemes for the delayed dynamical systems include the Galerkin projections [47] and the discretization 
approaches [48]. Based on these approximation schemes, the delayed dynamical system can be transformed into a high-dimensional ordinary differential system. Significant time-consumption will be involved in precisely obtaining the portfolios of global dynamics of such a high-dimensional system in the parameter and state spaces. In addition to the high time-cost on the computation of global dynamics, the delay-induced winding phenomenon in the identification of bifurcation will be another challenge. As reported in Ref. [49], the self-intersection phenomenon of phase trajectory can be delay-induced in the autonomous and delayed dynamical system, which will lead to the difficulties in distinguishing periodic response and identifying the bifurcation mechanism.

For the present autonomous and delayed governor system, the winding phenomena of periodic response are observed with certain delays and the bifurcation identification becomes a difficult task. To address this problem, a type of semi-analytical method, i.e., the time domain collocation method (TDCM) [50], is adopted to determine the bifurcation types of periodic response for the following reasons. First, the TDCM is easily implemented without the redundant process of formula derivation and the limitation of nonlinear terms of dynamical systems. Second, to the best of the authors' knowledge, this method has only been applied in the analysis of the low-dimensional dynamical systems with simple nonlinearity [50-52]. It is expected that this method will also be effective in solving the high-dimensional dynamical system, especially for the delayed dynamical system with complex nonlinearity. Based on these two reasons, the TDCM is chosen in this study to obtain the approximate analytical solution of periodic response and identify corresponding bifurcations.

The main contributions of this work can be briefly summarized in three aspects. 
Firstly, for the mechanical governor system, it is necessary to study the global dynamics of the system in the parameter and state spaces with inherent time delay for a better understanding of the system performance. Secondly, for the overall development of the delayed nonlinear dynamical systems, the exploration on the computation and bifurcation identification of global dynamical behaviors in this paper will provide a meaningful reference to the numerical simulation of complicated dynamical behaviour. Thirdly, the winding phenomena for periodic responses are observed and further discussed in the delayed governor system. It is found that the Poincaré section analysis may fail its effectiveness in distinguishing period property of periodic orbit in some cases.

The rest of this paper is organized as follows. The governing equations of the autonomous mechanical governor system with time delay are briefly introduced in Sec. 2. The methodologies used for computation and bifurcation identification of two-parameter dynamics of the mechanical governor system are presented in Sec. 3, including the numerical integration strategy of time-delayed system, determinate indicators of dynamical response, GPU parallel computing technique and bifurcation identification method of periodic response. Based on the above-mentioned methods, the classification of global dynamical behavior of the mechanical governor system with and without time delay is derived and shown in Sec. 4. In addition, the detailed analyses on the observed dynamical phenomena are also presented in Sec. 4. Finally, the conclusions are drawn in Sec. 5.

\section{Governing equations of the mechanical governor system}

As developed in Ref. [15], the governing equations of a type of mechanical governor system with reaction time-delay generated from the working principle of 
engines can be described by the following functional differential equations:

$$
\begin{aligned}
& \frac{d x}{d t}=y \\
& \frac{d y}{d t}=\left(e+p z^{2}\right) \sin x \cos x+d z^{2} \cos x-\sin x-b y \\
& \frac{d z}{d t}=q \cos x_{\tau}-Q
\end{aligned}
$$

where $x_{\tau}=x(t-\tau)$ and the detailed modelling process can be found in Ref. [15]. For the delayed governor system (1), one equilibrium can be easily determined as:

$$
\begin{aligned}
& x^{*}=\arccos \left(\frac{Q}{q}\right), \quad y^{*}=0, \\
& z^{*}=\sqrt{\frac{(q-e Q) \sqrt{q^{2}-Q^{2}}}{Q\left(p \sqrt{q^{2}-Q^{2}}+q d\right)}}
\end{aligned}
$$

By adopting the coordinate transformations $u=x-x^{*}, v=y-y^{*}, w=z-z^{*}$, Eq. (1) can be re-expressed as

$$
\begin{aligned}
& \stackrel{\&}{\&}=v \\
& \&=\left(a_{0}+a_{1} w+p w^{2} / 2\right) \sin \left(2 u+2 x^{*}\right)+\left(a_{2}+a_{3} w+d w^{2}\right) \cos \left(u+x^{*}\right)-\sin \left(u+x^{*}\right)-b v \\
& \&=q \cos \left(u_{\tau}+x^{*}\right)-Q
\end{aligned}
$$

where $a_{0}=\left(p z^{* 2}+e\right) / 2, a_{1}=p z^{*}, a_{2}=d z^{* 2}, a_{3}=2 d z^{*}$ and the equilibrium is then translated into the origin, i.e., $O(0,0,0)$.

For the mechanical governor system (3), the linear stability and local bifurcation behavior as well as the effect of time delay have been well investigated in a two-parameter plane, i.e., the $(b, Q)$ plane in Ref. [15]. As an extension of Ref. [15], this paper will investigate the global behavior of the time-delayed system and discuss the influence of time delay in the same two-parameter plane. More specifically, the system parameters are identical to Ref. [15], i.e., $p=0.04, d=0.09, e=0.4, q=2$. The $(b, Q)$ parameters are chosen as the primary bifurcation parameters with the 
ranges of $b \in[0,2], Q \in(0,2]$. The time delay is selected as an auxiliary bifurcation parameter and a set of discrete values, i.e., $\tau=0,1,2,3,4.0637988,5$ are considered. It should be mentioned that the 1:4 resonant double Hopf bifurcation of equilibrium arises with $\tau=4.0637988$ and the detailed analysis of this bifurcation can be found in Ref. [15].

For the sake of description, rewriting the governing Eq. (3) into a compact form yields

$$
\begin{cases}X^{\&}=F\left(\mu ; X, X_{\tau}\right), & t>0 \\ X(t)=\varphi(t), & t \in[-\tau, 0]\end{cases}
$$

where $X=(u, v, w)^{T}$ and $X_{\tau}=(u(t-\tau), v(t-\tau), w(t-\tau))^{T}$ represent the present states and the past states, respectively. The newly introduced symbol $\mu=\mu(b, Q, \tau)$ denotes the chosen bifurcation parameters and $F\left(\mu ; X, X_{\tau}\right)$ represents the right-hand functions of Eq. (3). In addition, $\varphi(t)$ is the continuous initial function of the delayed differential Eq. (3).

\section{Analysis methodologies}

\subsection{Numerical integration algorithm}

For the dynamical system with time delay and strong nonlinearity, the exact analytical solution is almost unavailable and the direct numerical integration becomes an indispensable technique to solve the complex functional differential equations. In this study, the fourth-order Runge-Kutta method with fixed step and the linear interpolation technique are combined to solve the delayed differential Eq. (4) with the initial value problem.

To begin with, the procedure of dimension reduction of the delayed governor system (4) is implemented via the discretization operation [48]. By dividing the initial 
interval $[-\tau, 0]$ into $N_{\tau}$ segments with $\left(N_{\tau}+1\right)$ time nodes, the discrete step size denoted by $\Delta t$ can then be calculated as $\Delta t=\tau / N_{\tau}$, where $N_{\tau}$ is a large positive integer. Based on the discretization operation, the continuous initial function $\varphi(t)$ can be approximated as an initial vector $Y_{0}$ :

$$
Y_{0}=[X(0) \mathrm{K} X(-i \cdot \Delta t) \mathrm{K} X(-\tau)]^{T}, \quad i=0,1, \mathrm{~K}, N_{\tau}
$$

The component $X\left(t_{i}\right)$ at the time instant $t_{i}=-i \cdot \Delta t$ represents $X\left(t_{i}\right)=\left(u_{i}, v_{i}, w_{i}\right)^{T}$. Let $h$ be the integral step size and satisfy the condition $h \leq \Delta t$, the single-step iteration map $\Phi$ can then be conducted as:

$$
Y_{h}=\Phi\left(Y_{0}, h\right)=\left[X^{\phi}(h) \mathrm{K} X^{\hat{\varphi}}(-i \cdot \Delta t+h) \mathrm{K} X^{\hat{\varphi}}(-\tau+h)\right]^{T}, \quad i=0,1, \mathrm{~K}, N_{\tau}
$$

By selecting the proper numerical integration algorithm, all components of the new iteration vector $Y_{h}$ can be expressed as:

$$
\left\{\begin{array}{l}
X(h)=X(0)+\frac{h}{6}\left(K_{1}+2 K_{2}+2 K_{3}+K_{4}\right), \\
X^{\prime}(-j \cdot \Delta t+h)=h \cdot[X((-j+1) \cdot \Delta t)-X(-j \cdot \Delta t)] / \Delta t+X(-j \cdot \Delta t), j=1, \mathrm{~K}, N_{\tau}
\end{array}\right.
$$

where

$$
\left\{\begin{array}{l}
K_{1}=F(\mu ; X(0), X(-\tau)) \\
K_{2}=F\left(\mu ; X(0)+0.5 h \cdot K_{1}, X(-\tau+0.5 h)\right) \\
K_{3}=F\left(\mu ; X(0)+0.5 h \cdot K_{2}, X(-\tau+0.5 h)\right) \\
K_{4}=F\left(\mu ; X(0)+h \cdot K_{3}, X(-\tau+h)\right)
\end{array}\right.
$$

and the component $X(-\tau+0.5 h)$ is obtained by using the linear interpolation, namely

$$
X(-\tau+0.5 h)=0.5 h \cdot[X(-\tau+\Delta t)-X(-\tau)] / \Delta t+X(-\tau)
$$

\subsection{Largest Lyapunov exponent and Poincaré section}

Lyapunov exponent is an effective indicator for the quantitative classification of different system responses. For an autonomous dynamical system, the system is in the 
state of equilibrium if the largest Lyapunov exponent $(L L E)$ is negative. Chaotic oscillation will appear in the system response if there exists a positive $L L E$. Otherwise, the system will exhibit periodic or quasi-periodic motions, if the $L L E$ equals to zero [53]. When the periodic and quasi-periodic motions need to be further distinguished, the second largest Lyapunov exponent or the Poincaré section will be examined. The $L L E$ has received the most attention for its high-efficiency in distinguishing different dynamical responses. In this paper, the estimation of $L L E$ is based on the method proposed in Ref. [54]. According to the estimation scheme [54] as shown in Fig. 1, the largest Lyapunov exponent is calculated by

$$
L L E=\lim _{n_{1} \rightarrow+\infty} \lim _{d_{0} \rightarrow 0} \frac{1}{n_{1} h} \sum_{i=1}^{n_{1}} \ln \frac{d_{i}}{d_{0}}
$$

where $h$ is the integral step size and $n_{1}$ is the total number of iterations. Symbol $d_{0}$ represents the initial distance between two nearby phase trajectories, i.e., $Y_{0}^{(0)}$ and $Y_{1}^{(0)} \cdot d_{i}$ represents the evolution distance between the two trajectories at the $i$ th iteration. In this scheme, renormalization is required for each iteration, namely $Y_{1}^{(i)}=d_{0} \cdot\left(Y_{1}^{(i)}-Y_{0}^{(i)}\right) / d_{i}$ would replace $Y_{1}^{(i)}$ as the new disturbed initial vector.

In addition to the Lyapunov exponents, the Poincare section is another widely used tool to distinguish the types of system responses. Generally, the system exhibits chaotic oscillation when a large number of disordered discrete points are found on the Poincaré section. The system will show quasi-periodic oscillation when a closed curve is observed on the Poincaré section. Periodic oscillation occurs in the system response when limited and countable points are observed on the section. However, it might be inaccurate to determine the period property of periodic response by only counting the points on the Poincare section for the autonomous system with time delay, since the self-intersection phenomenon of phase trajectory [49] may mislead the classification. 
To overcome this confusion, the other methods will also be applied and described in subsequent anlaysis. In this paper, the Poincaré section is selected as the section of zero angular velocity and given by:

$$
\Pi=\left\{\left(u_{0}, v_{0}, w_{0} \mathrm{~K} v_{N_{\tau}}, w_{N_{\tau}}\right) \in R^{3\left(N_{\tau}+1\right)} \mid v_{0}=0, \&>0\right\}
$$

It should be mentioned that the point of zero angular velocity on Poincaré section is detected from the binary process by varying integral step size $h$. Moreover, the Poincaré section $\Pi$ is mainly used for the classification of different periodic responses in two-parameter diagram and the presentation of single-parameter bifurcation diagram.

\subsection{GPU parallel computing}

Using the above mentioned numerical computation schemes, the single-parameter bifurcation diagram of the delayed mechanical governor system can be easily obtained. However, it is often a partial understanding of the delayed governor dynamics by only varying a single parameter. To explore the variation of the dynamical behavior of delayed dynamics with simultaneous changes of multiple system parameters, the GPU parallel computing technique is considered here to achieve the two-parameter diagram.

By dividing the two-parameter plane $(b, Q)$ into $L \times L$ grid points uniformly, $L \times L$ groups of independent cases can be obtained. Thus, the global dynamics can be further determined by solving the $L \times L$ deterministic governing equations. Benefiting from the NVIDIA company introduced CUDA-capable GPU parallel computing technique, this task can be implemented. In the present GPU parallel computing program, the total integration time $n_{1} h$ is set as 10000 , where $h$ is the integral step size and $n_{1}$ is the number of iteration. The first 3000 integration time is neglected as the elimination of transient effect and the subsequent 7000 integration 
time is applied to calculate the $L L E$. After that, a limited integration time is taken to calculate the points on Poincaré section and symbolized by $N i$, where $i$ is the number of intersection points between the phase trajectory and Poincaré section. By running the designed parallel computing program, the two indicators are recorded for each grid point and the category of system response can be accordingly achieved in the two-parameter plane. More specifically, the equilibrium point and chaos are first distinguished by the value of $L L E$, namely the equilibrium point is separated from the other system responses by condition $L L E<-\varepsilon$ and the chaos is detected by $L L E>\varepsilon$, where $\varepsilon$ is a user-defined small positive constant with $\varepsilon=0.001$ in this paper for the consideration of numerical integration error. Furthermore, the simple periodic response with $|L L E| \leq \varepsilon$ and $i \leq 6$ is characterized. For the convenience of distinction of all the presented colors, the rest system responses, e.g., the long periodic response with $i>6$ and the quasi-periodic response, are not specifically distinguished and represented by the same color.

\subsection{Time domain collocation method}

In the study, the time domain collocation method (TDCM) [50] is chosen as a theoretical tool to identify the bifurcation types of periodic response. The principle of TDCM will be briefly described in this subsection. Since the mechanical governor system (4) is autonomous and the frequency is unknown, the time re-scaling operation of Eq. (4) is conducted firstly for the convenience of the use of TDCM. Let $t_{1}=\Omega t, \delta=\Omega \tau$, then the time re-scaling governor system becomes:

$$
X^{\prime}=\frac{1}{\Omega} F\left(\mu ; X, X_{\delta}\right)
$$

where $X^{\prime}=d X / d t_{1}$ and $X_{\delta}=X\left(t_{1}-\delta\right)$. The period of system (11) has been transformed into $2 \pi$ at this time. Approximating the solution $X\left(t_{1}\right)$ of Eq. (11) by 
the Fourier series expansion yields:

$$
X\left(t_{1}\right)=A_{0}+\sum_{k=1}^{N} A_{2 k-1} \cos \left(k t_{1}\right)+A_{2 k} \sin \left(k t_{1}\right)
$$

where $A_{k}=\left(a_{k}^{(u)}, a_{k}^{(v)}, a_{k}^{(w)}\right)^{T}, k=0,1, \mathrm{~K}, 2 N$ are the $3 \cdot(2 N+1)$ undetermined coefficients and $N$ is the harmonic number in the truncated Fourier series solution. Correspondingly, the derivative and the delayed state can be expressed as:

$$
\begin{gathered}
X^{\prime}\left(t_{1}\right)=\sum_{k=1}^{N}\left[-k A_{2 k-1} \sin \left(k t_{1}\right)+k A_{2 k} \cos \left(k t_{1}\right)\right] \\
X\left(t_{1}-\delta\right)=A_{0}+\sum_{k=1}^{N} A_{2 k-1} \cos \left(k\left(t_{1}-\delta\right)\right)+A_{2 k} \sin \left(k\left(t_{1}-\delta\right)\right)
\end{gathered}
$$

Substituting Eq. (12)-Eq. (14) into the re-scaled governing Eq. (11) leads to the expression of the residual function:

$$
R\left(t_{1}\right)=\Omega X^{\prime}-F\left(\mu ; X, X_{\delta}\right)
$$

By dividing the period interval $[0,2 \pi]$ into $(M-1)$ segments, $M$ equidistant points $t_{1 j}, j=1,2, \mathrm{~K}, M$ are obtained. Thereafter, a series of residual functions for all the equidistant points are derived as:

$$
R_{j}\left(A_{0}, A_{1}, \mathrm{~K}, A_{2 N}\right)=\Omega X^{\prime}\left(t_{1 j}\right)-F\left(\mu ; X\left(t_{1 j}\right), X\left(t_{1 j}-\delta\right)\right)=\mathbf{0}
$$

To obtain the above $3 \cdot(2 N+1)+1$ unknowns, i.e., $3 \cdot(2 N+1)$ harmonic coefficients $A_{k}, k=0,1, \mathrm{~K}, 2 N$ and frequency $\Omega$, the number of algebraic Eq. (15) should be larger than the number of the unknowns. That is, the number of collocation points $M$ should satisfy the condition of $3 \cdot M>3 \cdot(2 N+1)+1$. For the consideration of the balance between the simulation accuracy and time consumption, the value of $M$ is determined by the specific case. The detailed description of the over-determined algebraic Eq. (15) can be found in Ref. [51]. After that, by numerically solving Eq. (15), the harmonic coefficient vector $A_{k}$ and the frequency 
$\Omega$ can be confirmed.

\subsection{Transition matrix for stability analysis}

To analyze the stability and bifurcation of the detected periodic response, the state transition matrix will be needed. Based on the obtained harmonic solution of system (11) and regarding it as the initial condition, the state transition matrix $G_{i}$ in single integral step can be theoretically determined as

$$
G_{i}=\left[\begin{array}{ccc}
\frac{\partial X^{\prime}(h)}{\partial X(0)} & \mathrm{L} & \frac{\partial X^{q}(h)}{\partial X(-\delta)} \\
\mathrm{M} & \mathrm{M} & \mathrm{M} \\
\frac{\partial X^{\prime}(-\delta+h)}{\partial X(0)} & \mathrm{L} & \frac{\partial X^{\prime}(-\delta+h)}{\partial X(-\delta)}
\end{array}\right]_{n\left(N_{\tau}+1\right) \times n\left(N_{\tau}+1\right)}
$$

where $N_{\tau}$ represents the segments derived from the equidistant division of the initial interval $[0,-\delta]$ and the discrete step size is denoted by $\Delta t=\delta / N_{\tau}$. The integral step size $h$ is given by $h=2 \pi / N_{T}$, where $N_{T}$ is the segments derived from the equidistant division of the period interval $[0,2 \pi]$. It should be mentioned that the integral step $h$ is set as $h \leq \Delta t$ for the convenience of calculation. Based on the numerical treatments shown in Eq. (7), the state transition matrix in a single integral step can be theoretically determined by Eq. (16).

By performing the multiplicative operation of $G_{i}, i=1, \mathrm{~K}, N_{T}$ in a period time, i.e., $[0,2 \pi]$, the state transition matrix $G$ of the periodic response yields [48]

$$
G=G_{N_{T}} G_{N_{T}-1} \mathrm{~L} G_{1}
$$

Accordingly, the stability and bifurcation of the periodic response can be determined by the eigenvalues of $G$, termed as the Floquet multipliers. From Ref. [55], it is known that there are $n$ Floquet multipliers for the $n$-dimensional dynamical system and one of them called the trivial Floquet multiplier always equals to the constant 1 . 
The stability and bifurcation of periodic orbit can be determined by the distribution of the rest $(n-1)$ nontrivial Floquet multipliers [55].

\section{Two-parameter dynamics of the delayed governor system}

\subsection{Motion classification in the $(b, Q)$ two-parameter plane}

In the present work, the $(b, Q)$ two-parameter plane is uniformly divided into $500 \times 500$ grid points for the sake of reducing computation time. By simulating $500 \times 500$ deterministic governing differential Eq. (3), the two indicators, i.e., the $L L E$ and the number of intersection points on Poincaré section, are calculated and recorded. After that, the motion classification of the mechanical governor system can be obtained in terms of the two indicators, and the results are shown in Figs. 2(a-f). In Figs. 2(a-f), ten colors are used and the corresponding colored bars are presented in Fig. 2(g). Here, symbol $D$ indicates the diverging response, symbol $C$ denotes the chaotic motions, symbol $E$ indicates the equilibrium state, $N 1: N 6$ correspond to the simple periodic responses and $N Q$ represents the long periodic motions with $N 7, N 8, \mathrm{~K}$ types and quasi-periodic motions.

A comparison of the results shown in Figs. 2(a-f) with those in Ref. [15] shows that the stable regions of equilibrium and its boundaries are identical to each other. Additionally, it has been reported in Ref. [15] that the equilibrium would lose its stability mainly through Hopf bifurcation. The bifurcating limit cycle (indicated by symbol NI and pink regions) are also identified near the stability boundaries of the equilibrium states, as shown in Figs. 2(a-f). Moreover, the fine structures lying on the left bottom of the two-parameter plane and the steady-state response originating from a certain co-dimension two bifurcation of equilibrium, which have been reported in Ref. [15], are also detected and shown in Figs. 2(a-f). These features indicate the 
effectiveness of the numerical computation strategies designed in this paper.

For the nonlinear dynamics uncovered in Ref. [15], the numerical results presented in Figs. 2(a-f) can now provide a clear interpretation and bring new insights. For example, the N2: N6 types of periodic responses and chaotic motions revealed here have not been reported in Ref. [15]. In the parameter region of chaotic motions in Figs. 2(a-c), the existence region of such motion shrinks when the time delay varies from 0 to 2. Specifically, no chaos region is found in the two-parameter diagram in Fig. 2(c) with $\tau=2$ and the stable region of equilibrium marked by the gray shadow is divided into two parts in this case. To clarify this "break" phenomenon, the effect of time delay on the evolution of stability boundary of the equilibrium is further investigated. It is found that the observed "break" phenomenon is caused by the range limitation of parameter $b$, rather than by any novel bifurcations of the equilibrium. To better illustrate such phenomenon, the stability boundary of equilibrium with $\tau=1.7$ is plotted in Fig. 3. As indicated in Fig. 3, the left part of the vertical black solid line is the interested region under the consideration of nonlinear dynamics analysis and the right part is not considered in the study. Besides, although no chaotic response occurs in the system with $\tau=2$ in Fig. 2(c), the existence region of chaotic motion would emerge again with the increase of time delay, as can be seen from Figs. 2(d-f)

In addition to chaotic motion, different periodic responses are also revealed in the two-parameter region, where the equilibrium loses its stability. According to the distributions of periodic response, it seems that the transitions of periodic responses become more and more complicated with the increase of time delay, especially for the cases of Figs. 2(e-f). It should be noted that the boundaries of different colors shown in Figs. 2(a-f) do not indicate the actual bifurcation curves. To determine the 
bifurcations shown in Figs. 2(a-f), the single-parameter bifurcation analysis would be required for better understanding of the obtained two-parameter results.

\subsection{Single-parameter bifurcation analysis}

\subsubsection{Continuation analyses for the cases of $\tau=0$ and $\tau=1$}

For the case of $\tau=0$ in Fig. 2(a), the single-parameter bifurcation diagrams are obtained by using the direct numerical integration (DNI) and path-following strategy. The corresponding results in terms of the chosen Poincaré section determined by Eq. (10) are marked by the blue dots and shown in Figs. 4(a-b). It can be seen that there exist two types of jump phenomena in these diagrams, i.e., the jump between two branches of stable $N 1$ type of periodic response and the jump between $N 3$ type of periodic response and chaos. To conduct the continuation analysis and reveal the transitions of stabilities, the time domain collocation method (TDCM) and the transition matrix mentioned in Sec. 3.4 and Sec. 3.5 are applied to identify the bifurcations. Firstly, for the jump between two branches of stable N1 type of periodic response in Figs. 4(a-b), the unstable solutions are tracked and marked by the red dotted lines. It is found that such jump phenomena are mainly caused by the saddle-node (SN) bifurcations of $N 1$ type of periodic response. Secondly, for the jump between $N 3$ type of periodic response and chaotic motion, the continuation analysis is also implemented to investigate the generation mechanism of the $N 3$ type of periodic solution. For the case shown in Fig. 4(a), the appearance and disappearance of N3 type of periodic response are both induced by the SN bifurcation. However, some coexisting attractors can be newly discovered and displayed in the inserted windows in Fig. 4(b). These newly detected attractors might be often neglected if the continuation analysis of $\mathrm{N} 3$ type of periodic response is not fully considered. By increasing the bifurcation parameter $b$ of Fig. 4(b), the stable $N 3$ type of periodic 
response shall lose its stability through the subcritical period-doubling (PD) bifurcation on the red dotted line, as shown in the inserted windows. The unstable $N 3$ type of periodic solution marked by the red dots appears accordingly. Thereafter, the unstable $N 3$ type of periodic solution will regain its stability by a supercritical PD bifurcation. What should be noted is that chaotic attractor will appear near such a supercritical PD bifurcation. And the newly stable $N 3$ type of periodic response will finally disappear via an SN bifurcation.

For the case of $\tau=1$ of Fig. 2(b), the single-parameter bifurcation diagrams and the continuation results are presented in Figs. 5(a-b). From Fig. 5(b), it can be seen that a typical PD bifurcation route from periodic solution to chaotic solution is observed. Therefore, the focus will be put on the jump phenomena in Fig. 5(a). Different from the jump mechanism of $N 1$ type of periodic response revealed in Fig. 4(a), the case in Fig. 5(a) is more complicated. Two branches of unstable periodic solutions, i.e., branches 1 and 2 , are traced. To better illustrate the evolutionary process in the case, variation tendencies of the largest two Floquet multipliers with the change of parameter $Q$ are presented in Fig. 5(c-d). Here, the trivial Floquet multiplier 1 (which always exists) is not displayed in the diagrams. Apart from this trivial multiplier 1, the rest multipliers are ranked and the top two multipliers, i.e., $\lambda_{1}, \lambda_{2}$ with $\left|\lambda_{1}\right| \geq\left|\lambda_{2}\right|$, are displayed. The ranked multipliers corresponding to the branch 1 are shown in Fig. 5(c), while those of branch 2 are shown in Fig. 5(d). It can be seen that a limit point exists at the intersection point between these two branches, since a nontrivial Floquet multiplier +1 is calculated and the largest Floquet multiplier is larger than 1 , i.e., $\lambda_{1}>1, \lambda_{2} \approx+1$. Besides, one pair of purely imaginary eigenvalues are calculated in Fig. 5(d), which indicates a NS bifurcation of branch 2 . As for the jump between $N 3$ type of periodic motion and chaos in Fig. 5(a), similar 
scenarios to Fig. 4(b) are found and the newly bifurcating system responses are shown in the inserted windows.

From the above analyses, rich phenomena of multistability are observed in the system response, such as the coexistence of periodic response and chaotic motion, and coexistence of varieties of periodic responses. The details on these coexisting response will be discussed later.

\subsubsection{Winding phenomena and bifurcations with $\tau \geq 2$}

When increasing the time delay to $\tau=2$, the single-parameter bifurcation diagrams are plotted in Figs. 6(a-b). It is easy to notice that a supercritical PD bifurcation will arise in these cases. And the system will exhibit simple behaviors, e.g., the equilibrium state and simple periodic oscillations. When the time delay is further increased to $\tau=3$, the single-parameter bifurcation diagrams shown in Figs. 7(a-b) become more complicated and the transition mechanisms become hard to be identified. From Figs. 7(a-b), it seems that a supercritical PD bifurcation occurs in these cases, while some excess responses would exist along the supercritical PD bifurcation tree. To verify the evolutionary process in these cases, a set of phase diagrams are inserted into Figs. 7(a-b). From the inserted diagrams, it can be seen that the winding phenomena of phase trajectories appear, i.e., the period property of periodic responses cannot be clearly determined by the number of intersection points of the Poincaré section.

To address this problem, the TDCM will be applied to obtain the analytical solutions of the complex periodic orbits and further clarify the bifurcation types.

Taking region I (black dotted circle) in Fig. 7(a) as an example, $N=12$ is chosen in the Fourier series expansion, i.e., Eq. (12), and the number of collocation points $M$ is set as $M=34$ in this case. Accordingly, the analytical solutions of the 
periodic responses corresponding to region I can be derived and marked as red circles in Fig. 7(c). The relevant largest two Floquet multipliers are exhibited in Fig. 7(d). From Fig. 7(c), it can be seen that the theoretical results are in a good agreement with the numerical results. It is also noticed that the PD bifurcation occurs on the red dotted line and no bifurcations are detected on the black dotted lines by analyzing the Floquet multipliers in Fig. 7(d). To explore the transition mechanism of just beforeand after- the black dotted lines in Fig. 7(c), the relationship between the bifurcation parameter and the harmonic components of analytical solutions, i.e., the harmonic orders and amplitudes, is studied next.

To begin with, re-expressing the harmonic amplitudes in Eq. (12) as

$$
\begin{aligned}
& c_{i, 0}=a_{0}^{(i)}, \\
& c_{i, k}=\sqrt{a_{2 k-1}^{(i) 2}+a_{2 k}^{(i) 2}}, \quad k=1, \mathrm{~K}, N
\end{aligned}
$$

where $i=1,2,3$ corresponds to the state variables $u, v, w$, respectively. The symbol $k$ represents the $k$ th harmonic order. For the periodic responses shown in Fig. 7(c), the distribution of points on Poincar é section varies in the form of $N 1 \rightarrow N 2 \rightarrow N 3 \rightarrow N 2$. The variations of all thirteen harmonic components in the analytical solutions, i.e., $k=0, \mathrm{~K}, 12$, with respect to the bifurcation parameter $Q$ are demonstrated in Figs. 7(e-f), where the harmonic components for the state $u$ are displayed in Fig. 7(e) and harmonic components for the state $v$ are shown in Fig. 7(f). It is noted that only the first few harmonic components are marked by $c_{i, k}$ in Figs. 7(e-f) for their significance in characterizing the periodic orbits. By comparing the harmonic components before- and after- the critical lines represented by the back dotted lines, it is found that the change in the number of intersection points on Poincar é section is caused by the varying harmonic amplitudes rather than the bifurcation. Besides, the fact that the existence of even harmonic components in the analytical 
solutions can induce the asymmetry of periodic orbits [56] is also witnessed in this analysis. Same as the bifurcation identification procedure for the complex periodic response in Fig. 7(a), the analytical solutions and the Floquet multipliers are also calculated for the case of Fig. 7(b), and a PD bifurcation is determined in this case. Furthermore, the bifurcation types of periodic orbits along the chosen bifurcation lines for the cases of $\tau=4.0637988$ and $\tau=5$ in Figs. 2(e-f) are clarified accordingly.

For the case of $\tau=4.0637988$, the corresponding single-parameter bifurcation diagrams are shown in Figs. 8(a-b). By comparing the evolutionary process of Figs. 8(a-b) with Figs. 7(a-b), the asymmetry feature of the periodic orbits becomes more and more complicated as time delay increases. To identify the bifurcation mechanisms in Figs. 8(a-b), the Floquet multipliers are calculated and the involved bifurcation types are indicated in the two diagrams. Specifically, the identifications of bifurcations by checking the Floquet multipliers in regions II and III of Fig. 8(a) are presented in Figs. 8(c-e), respectively. In region II, the $N 3$ type of periodic response loses its stability by the subcritical NS bifurcation, as one pair of conjugate complex Floquent multipliers with modulus 1 are detected in Fig. 8(c). Meanwhile, the coexistence of attractors can be observed in this region, as shown in the inserted phase diagram. For the bifurcations in region III, the N1 and N3 types of periodic responses will both disappear by the SN bifurcation, since the approximated Floquent multiplier +1 can be predicted in Fig. 8(d-e). In region Ш, the coexistence of these independent periodic solutions can be observed and the coexisting attractors are shown in the inserted phase portrait of Fig. 8(a). For the bifurcation diagram shown in Fig. 8(b), the supercritical PD bifurcation route arises. However, the evolutionary process of periodic responses shown in Fig. 8(b) is obviously more complicated than the case in Fig. 7(b). 
For the case of $\tau=5$, the resultant single-parameter bifurcation diagrams are displayed in Figs. 9(a-b). As mentioned previously, the evolutionary process in this case is much more complicated than that in Figs. 8(a-b). That is, the relationship between the distribution of intersection points on the chosen Poincaré sections and periodic characteristic becomes more indistinguishable. To distinguish different responses and clarify bifurcation behaviors in these cases, the Floquet multipliers are also calculated and the corresponding bifurcation types are inserted in Figs. 9(a-b). The PD bifurcation is the main bifurcation type in these two cases. Specifically, a supercritical NS bifurcation tree is found in Fig. 9(a), which coexists with a PD bifurcation tree. Accordingly, the multistabilities, i.e., periodic response coexisting with quasi-periodic response, can be observed. Moreover, the coexistence pattern of two independent periodic responses is detected in region IV of Fig. 9(a) and will be discussed in the following subsection.

\subsubsection{Co-dimension two bifurcation of equilibrium for $\tau=5$}

A co-dimension two Hopf bifurcation point of equilibrium could be found in the neighborhood (in terms of parameters) of region IV of Fig. 9(a) and has been reported in Ref. [15]. Based on the results of Ref. [15], four types of responses will arise in region IV, i.e., equilibrium $E_{0}$, two periodic solutions $E_{1}$ and $E_{2}$, and one unstable quasi-periodic solution $E_{3}$. The bifurcations and transitions between the four responses are schematically presented in Fig. 9(c). Using the DNI and path-following methods, the steady response of system in region IV is traced and indicated by blue dots in Fig. 9(d). It can be seen that the transitions of Fig. 9(d) are in accordance with the transitions marked by the black solid lines in Fig. 9(c). As for the jump phenomenon arising between $E_{1}$ and $E_{2}$ in Fig. 9(c), it is also verified by numerical results in Fig. 9(d). 
To verify the transition mechanisms in terms of the periodic solutions $E_{1}$ and $E_{2}$, the analytical solutions are determined as the red circles shown in Fig. 9(d). The upper branch of analytical solutions marked by intensive red circles is derived by tracing the periodic solution $E_{1}$. The lower branch of analytical solutions marked by sparse red circles is derived by tracing the periodic solution $E_{2}$. For the upper branch $E_{1}$, one pair of purely imaginary Floquet multipliers $\lambda_{1,2} \approx-0.560 \pm 0.858 i$ can be calculated on line $l_{3}$ and periodic solution $E_{1}$ will become unstable via the subcritical NS bifurcation. Furthermore, the unstable periodic solution $E_{1}$ will disappear upon passing line $l_{2}$ and one limit nontrivial Floquet multiplier +1 arise. For the lower branch $E_{2}$, the $\mathrm{SN}$ bifurcation occurs on line $l_{1}$ and then $E_{2}$ disappears.

\subsection{Multistable phenomena and chaos routes}

This subsection discusses the qualitative or quantitative changes of the system responses in the state plane. As mentioned previously, a number of coexisting attractors has been observed in the mechanical governor system with and without time delay. For the non-delay cases shown in Figs. 4(a-b), two main bifurcation trees are observed under different initial perturbations. One bifurcation tree originates from the supercritical PD bifurcation of $N 1$ type of periodic response and the other is caused by the supercritical PD bifurcation of $N 3$ type of periodic response. Accordingly, rich coexistence phenomena can be induced in the governor system without time delay, such as the coexistence of different periodic attractors, coexistence of different periodic attractors and chaos. In addition to the above-mentioned coexistence patterns, the coexistence of equilibrium and periodic response can also be observed in this case.

To study the evolutionary process of basins of attraction, the coexistence of $\mathrm{N} 3$ 
type of periodic response and chaos arising in Fig. 4(a) is taken as an illustrative example. Four groups of parameters are selected to compute the basins of attraction and the results are presented in Figs. 10(a-d) accordingly. The basin of attraction is also obtained by the GPU parallel computing technique. The state space is considered as follows,

$$
\Pi_{1}=\left\{(u, v, w) \in R^{3} \mid-5 \leq u \leq 5, v=0,-5 \leq w \leq 5\right\}
$$

And $\Pi_{1}$ is divided into $300 \times 300$ grid points in the parallel computing program for the consideration of balance between computational accuracy and time consumption. In Figs. 10(a-d), the red domain indicates the basin of attraction of $N 3$ type of periodic response, while the dark green domain stands for the basin of attraction of chaos. And the rest rufous domain corresponds to the diverging response. By analyzing the basins of attraction shown in Figs. 10(a-d), it can be seen that the phenomenon of self-similarity structure arises. The basin of attraction of the $N 3$ type of periodic response gradually shrinks and the basin of attraction of chaos will expand when the bifurcation parameter $Q$ increases. Eventually, the basin of attraction of $N 3$ periodic motion disappears through an SN bifurcation, as indicated in Fig. 4(a).

For the mechanical governor system with time delay, some coexistence phenomena are newly found. When the time delay is small as the case shown in Fig. 5(a), a new coexistence of stable $N 1$ type and $N 3$ type of periodic responses emerges. As the time delay increases, new dynamic phenomena are observed, e.g., the coexistence of two branches of NS bifurcation tree in region II of Fig. 8(a) and the co-dimension two bifurcation of equilibrium in Fig. 9(a). Accordingly, two coexistence patterns, i.e., coexistence of $N 1$ type and $N 3$ type of periodic attractors, coexistence of periodic and quasi-periodic attractors, occur in the cases of Fig. 8(a) and Fig. 9(a), respectively. To illustrate the coexistence of the $N 1$ type and $N 3$ type of 
periodic attractors for the case of Fig. 8(a), the basin of attraction is considered to be computed in the following sate space:

$$
\Pi_{2}=\left\{\left(u_{i}, v_{i}, w_{i}\right) \in R^{3\left(N_{\tau}+1\right)} \mid-5 \leq u_{0} \leq 5, v_{0}=0,-5 \leq w_{0} \leq 5, u_{i}=u_{0}, v_{i}=v_{0}, w_{i}=w_{0}\right\}
$$

where $i=1, \mathrm{~K}, N_{\tau}$. In the case, the state plane $\left(u_{0}, w_{0}\right)$ is displayed and also divided into $300 \times 300$ grid points. Moreover, four groups of parameters are chosen to study the evolution of the basin of attraction. The corresponding results are exhibited in Fig. 11 , where the red domain is the basin of attraction of the $N 3$ type of periodic response, and the green domain represents the basin of attraction of $N 1$ type of periodic response. The rest rufous domain corresponds to the diverging motion under the chosen initial condition. Same as the case in Fig. 10, the self-similar basin structure is also observed in Fig. 11. Due to the range limitation of the selected state plane, the self-similar basin structures located on the right hand sides of Figs. 11(a-d) are partially presented and would not be further studied in this paper. It is easy to find that the evolution tendency of the basin of attraction of the two different periodic solutions is identical with the bifurcation diagram in Fig. 8(a).

In the chaotic transitions exhibited in the above results, three main routes leading to chaos can be found in the system response. The first route is that the chaotic motion is generated from a PD bifurcation and then disappears by the way of boundary crisis [57], e.g., Figs. 4(a-b) and Fig. 5(a). In these cases, the PD bifurcation is involved with the supercritical type. The second route is that the chaotic motion is born and destroyed both via a PD bifurcation, e.g., Fig. 4(b), Fig. 5(b) and Fig. 7(a). The third route is that the chaotic motion arises from a NS bifurcation, such as Fig. 8(a). In Fig. 8(a), the subcritical NS bifurcation of N1 type of periodic response is involved and such a route has ever been reported in Ref. [57].

\section{Conclusion}


To explore the global behavior of the governor dynamics with and without time delay, a two-parameter dynamic analysis has been carried out by using the CUDA-capable GPU computing technique. The uncovered dynamics in Ref. [15] has been systematically investigated by the single-parameter bifurcation diagram and path-following technique. It was found that the complex periodic orbits could arise in the governor system with the increase of time delay, which would cause the failure of Poincaré section analysis in the identification of bifurcations. To address this problem, the analytical approach based on the time domain collocation method has been further applied to clarify the bifurcation mechanisms. A number of multistability patterns has been found in the dynamical behavior of the system with and without time delay, and the basins of attraction presented correspondingly. Meanwhile, the observed chaotic transitions were discussed and summarized.

Based on the work presented in Ref. [15] and the present study, the two-parameter dynamics of the delayed governor system has been extensively investigated. It is anticipated that these research results would provide a meaningful reference to the optimal design and motion control of the mechanical systems and the exploration of local and global dynamics of the delayed systems.

\section{Declarations}

\section{Funding}

This work has been supported by the Key Program of National Natural Science Foundation of China (No. 11832009), and the Applied Basic Research Program of Shanxi Province of China (No. 201801D121021). 


\section{Conflicts of interest}

The authors declare that they have no conflict of interest.

\section{Availability of data and material}

Data will be made available on reasonable request.

\section{References}

[1] Chen, Y., Cheng, J., Jiang, Y., Liu, K.: A time delay dynamical model for outbreak of 2019-nCoV and the parameter identification. J. Inverse Ill-Posed Probl. 28(2), 243-250 (2020).

[2] Zhang, X., Liu, Z.: Periodic oscillations in HIV transmission model with intracellular time delay and infection-age structure. Commun. Nonlinear Sci. Numer. Simul. 91, 105463 (2020).

[3] Khajanchi, S., Nieto, J.J.: Mathematical modeling of tumor-immune competitive system, considering the role of time delay. Appl. Math. Comput. 340, 180-205 (2019). [4] Li, L., Zhang, C.H., Yan, X.P.: Stability and Hopf bifurcation analysis for a two-enterprise interaction model with delays. Commun. Nonlinear Sci. Numer. Simul. 30(1-3), 70-83 (2016).

[5] Mutingi, M., Mbohwa, C., Kommula, V.P.: System dynamics approaches to energy policy modelling and simulation. Energy Procedia, 141, 532-539 (2017).

[6] Cheng, Z., Xie, K., Wang, T., Cao, J.: Stability and Hopf bifurcation of three-triangle neural networks with delays. Neurocomputing, 322, 206-215 (2018).

[7] Ma, J., Tang, J.: A review for dynamics in neuron and neuronal network. 
Nonlinear Dyn. 89(3), 1569-1578 (2017).

[8] Wu, K.N., Na, M.Y., Wang, L., Ding, X., Wu, B.: Finite-time stability of impulsive reaction-diffusion systems with and without time delay. Appl. Math. Comput. 363, 124591 (2019).

[9] Tan, Y., Huang, C., Sun, B., Wang, T.: Dynamics of a class of delayed reaction-diffusion systems with Neumann boundary condition. J. Math. Anal. Appl. 458(2), 1115-1130 (2018).

[10] Beregi, S., Takacs, D., Stepan, G.: Bifurcation analysis of wheel shimmy with non-smooth effects and time delay in the tyre-ground contact. Nonlinear Dyn. 98(1), $841-858$ (2019).

[11] Ji, J.C., Zhou, J.: Coexistence of two families of sub-harmonic resonances in a time-delayed nonlinear system at different forcing frequencies. Mech. Syst. Signal Proc. 93, 151-163 (2017).

[12] Zhang, C.H., He, Y.: Multiple stability switches and Hopf bifurcations induced by the delay in a Lengyel-Epstein chemical reaction system. Appl. Math. Comput. 378, $125201(2020)$.

[13] Yan, X.P., Liu, F.B., Zhang, C.H.: Multiple stability switches and Hopf bifurcation in a damped harmonic oscillator with delayed feedback. Nonlinear Dyn. 99(3), 2011-2030 (2020).

[14] Cantis á n, J., Coccolo, M., Seoane, J.M., Sanju án, M.A.: Delay-induced resonance in the time-delayed duffing oscillator. Int. J. Bifurcation Chaos 30(03), 2030007 (2020).

[15] Deng, S., Ji, J., Wen, G., Xu, H.: Delay-induced novel dynamics in a hexagonal centrifugal governor system. Int. J. Non-Linear Mech. 103465 (2020).

[16] Scholl, T.H., Gröll, L., Hagenmeyer, V.: Time delay in the swing equation: A 
variety of bifurcations. Chaos 29(12), 123118 (2019).

[17] Mao, X., Zhou, X., Shi, T., Qiao, L.: Dynamical analysis of coupled bidirectional FitzHugh-Nagumo neuronal networks with multiple delays. J. Comput. Nonlinear Dyn. 14(6) (2019).

[18] Yan, Y., Zhang, S., Guo, Q., Xu, J., Kim, K.C.: Energy determines multiple stability in time-delayed systems. Nonlinear Dyn. 102(4), 2399-2416 (2020).

[19] Yao, S., Ding, L., Song, Z., Xu, J.: Two bifurcation routes to multiple chaotic coexistence in an inertial two-neural system with time delay. Nonlinear Dyn. 95(2), 1549-1563 (2019).

[20] Gökçe, A., Yazar, S., Sekerci, Y.: Delay induced nonlinear dynamics of oxygen-plankton interactions. Chaos Solitons Fractals 141, 110327 (2020).

[21] Jin, Y., Meng, J.: Dynamical analysis of an optimal velocity model with time-delayed feedback control. Commun. Nonlinear Sci. Numer. Simul. 105333 (2020).

[22] Zhai, C., Wu, W.: Designing continuous delay feedback control for lattice hydrodynamic model under cyber-attacks and connected vehicle environment. Commun. Nonlinear Sci. Numer. Simul. 105667 (2020).

[23] Liu, J., Hu, B., Yang, F., Zang, C., Ding, X.: Stochastic Resonance in a Delay-controlled Dissipative Bistable Potential for Weak Signal Enhancement. Commun. Nonlinear Sci. Numer. Simul. 105245 (2020).

[24] Firoozy, P., Friswell, M. I., Gao, Q.: Using time delay in the nonlinear oscillations of magnetic levitation for simultaneous energy harvesting and vibration suppression. Int. J. Mech. Sci. 163, 105098 (2019).

[25] Yang, T., Cao, Q.: Time delay improves beneficial performance of a novel hybrid energy harvester. Nonlinear Dyn. 96(2), 1511-1530 (2019). 
[26] Zhang, X., Xu, J., Ji, J.: Modelling and tuning for a time-delayed vibration absorber with friction. J. Sound Vibr. 424, 137-157 (2018).

[27] Fu, J., Dai, Z., Yang, Z., Lai, J., Yu, M.: Time delay analysis and constant time-delay compensation control for MRE vibration control system with multiple-frequency excitation. Smart Mater. Struct. 29(1), 014001 (2019).

[28] Zhang, J.G., Mello, L.F., Chu, Y.D., Li, X.F., An, X.L.: Hopf bifurcation in an hexagonal governor system with a spring. Commun. Nonlinear Sci. Numer. Simul. 15(3), 778-786 (2010).

[29] Alidousti, J., Eskandari, Z.: Dynamical behavior and Poincaré section of fractional-order centrifugal governor system. Math. Comput. Simul. 182, 791-806 (2021).

[30] Zhang, R., Wang, Y., Zhang, Z., Bi, Q.: Nonlinear behaviors as well as the bifurcation mechanism in switched dynamical systems. Nonlinear Dyn. 79(1), 465-471 (2015).

[31] Marcondes, D.W.C., Comassetto, G.F., Pedro, B.G., Vieira, J.C.C., Hoff, A., Prebianca, F., Manchein, C., Albuquerque, H.A.: Extensive numerical study and circuitry implementation of the Watt governor model. Int. J. Bifurcation Chaos 27(11) 1750175 (2017).

[32] Rosa, L.A., Prebianca, F., Hoff, A., Manchein, C., Albuquerque, H.A.: Characterizing the Dynamics of the Watt Governor System Under Harmonic Perturbation and Gaussian Noise. Int. J. Bifurcation Chaos 30(01), 2030001 (2020).

[33] Yan, B., He, S., Sun, K., Wang, S.: Complexity and Multistability in the Centrifugal Flywheel Governor System With Stochastic Noise. IEEE Access, 8, 30092-30103 (2020).

[34] Rao, X.B., Chu, Y.D., Chang, Y.X., Zhang, J.G.: Broken Farey tree and fractal in 
a hexagonal centrifugal governor with a spring. Chaos Solitons Fractals 107, 251-255 (2018).

[35] Rao, X.B., Chu, Y.D., Chang, Y.X., Zhang, J.G.: Fractal structures in centrifugal flywheel governor system. Commun. Nonlinear Sci. Numer. Simul. 50, 330-339 (2017).

[36] Ge, Z.M., Jhuang, W.R.: Chaos, control and synchronization of a fractional order rotational mechanical system with a centrifugal governor. Chaos Solitons Fractals 33(1), 270-289 (2007).

[37] Ge, Z.M., Lee, C.I.: Control, anticontrol and synchronization of chaos for an autonomous rotational machine system with time-delay. Chaos Solitons Fractals 23(5), 1855-1864 (2005).

[38] Deng, S., Ji, J., Yin, S., Wen, G.: Multistability in the Centrifugal Governor System Under a Time-Delay Control Strategy. Comput. Nonlinear Dyn. 14(11) (2019).

[39] Song, Z., Sun, K., Ling, S.: Stabilization and synchronization for a mechanical system via adaptive sliding mode control. ISA Trans. 68, 353-366 (2017).

[40] Aghababa, M.P., Aghababa, H.P.: Finite-time stabilization of non-autonomous uncertain chaotic centrifugal flywheel governor systems with input nonlinearities. J. Vib. Control 20(3), 436-446 (2014).

[41] Wen, G., Xu, H., Lv, Z., Zhang, S., Wu, X., Liu, J., Yin, S.: Anti-controlling Hopf bifurcation in a type of centrifugal governor system. Nonlinear Dyn. 81(1-2), 811-822 (2015).

[42] Lv, Z., Xu, H., Bu, Z.: Control of Neimark-Sacker bifurcation in a type of weak impulse excited centrifugal governor system. Int. J. Non-Linear Mech. 128, 103624 (2021). 
[43] Ploskas, N., Samaras, N.: GPU programming in MATLAB. Morgan Kaufmann, Cambridge (2016).

[44] Yin, S., Wen, G., Ji, J., Xu, H.: Novel two-parameter dynamics of impact oscillators near degenerate grazing points. Int. J. Non-Linear Mech. 120, 103403 (2020).

[45] Rao, X.B., Chu, Y.D., Chang, Y.X., Zhang, J.G., Tian, Y.P.: Dynamics of a cracked rotor system with oil-film force in parameter space. Nonlinear Dyn. 88(4), 2347-2357 (2017).

[46] Klapcsik, K., Hegedüs, F.: The effect of high viscosity on the evolution of the bifurcation set of a periodically excited gas bubble. Chaos Solitons Fractals 104, 198-208 (2017).

[47] Wahi, P., Chatterjee, A.: Galerkin projections for delay differential equations. J. Dyn. Syst., Meas., Control 127(1), 80-87 (2005).

[48] Insperger, T., St é pá n, G.: Semi-discretization stability and engineering applications for time-delay systems. Springer, New York (2011).

[49] Wang, H., Hu, H.: Bifurcation analysis of a delayed dynamic system via method of multiple scales and shooting technique. Int. J. Bifurcation Chaos 15(2), 425-450 (2005).

[50] Dai, H.H., Schnoor, M., Atluri, S.N.: A simple collocation scheme for obtaining the periodic solutions of the duffing equation, and its equivalence to the high dimensional harmonic balance method: subharmonic oscillations. CMES-Comp. Model. Eng. Sci. 84(5), 459 (2012).

[51] Dai, H.H., Yue, X.K., Yuan, J.P.: A time domain collocation method for obtaining the third superharmonic solutions to the Duffing oscillator. Nonlinear Dyn. 73(1-2), 593-609 (2013). 
[52] Dai, H., Yue, X., Yuan, J., Atluri, S.N.: A time domain collocation method for studying the aeroelasticity of a two dimensional airfoil with a structural nonlinearity. J. Comput. Phys. 270, 214-237 (2014).

[53] Stefanski, A., Dabrowski, A., Kapitaniak, T.: Evaluation of the largest Lyapunov exponent in dynamical systems with time delay. Chaos Solitons Fractals 23(5), 1651-1659 (2005).

[54] Benettin, G., Galgani, L., Strelcyn, J.M.: Kolmogorov entropy and numerical experiments. Phys. Rev. A 14(6), 2338 (1976).

[55] Seydel, R.: Practical bifurcation and stability analysis. Springer, New York (2009).

[56] Zou, K., Nagarajaiah, S.: An analytical method for analyzing symmetry-breaking bifurcation and period-doubling bifurcation. Commun. Nonlinear Sci. Numer. Simul. 22(1-3), 780-792 (2015).

[57] Ott, E.: Chaos in dynamical systems. Cambridge university press, Cambridge (2002).

\section{List of Figure Captions}

Fig. 1 The schematic of estimation of the largest Lyapunov exponent.

Fig. 2 Motion distribution in the two-parameter $(b, Q)$ plane with different time delays. (a) $\tau=0$, (b) $\tau=1$, (c) $\tau=2$, (d) $\tau=3$, (e) $\tau=4.0637988$, (f) $\tau=5$, where the black asterisk denotes a co-dimension two bifurcation point of equilibrium with $(b, Q)=(0.2263231,0.7724652)$ and the details on such bifurcation can be found in Ref. [15], (g) Colorbar for different system responses: $D$-divergence; $C$-chaos; $E$-stable equilibrium; $N i, i=1: 6$-simple periodic response, where $i$ 
represents the number of intersection points between the Poincaré section and the phase trajectory; $N Q$-Complication of periodic response with $i>6$ and quasi-periodic response.

Fig. 3 Stability boundary of equilibrium with $\tau=1.7$.

Fig. 4 Single-parameter bifurcation diagrams along the black solid lines in Fig. 2(a). The blue sets represent the stable system responses which are derived from the direct numerical integration. The red sets represent the unstable system responses which are derived from the time domain collocation method. (a-b) the case of $\tau=0$ with $b=0.2$ and $Q=0.6$, respectively.

Fig. 5 Bifurcation analyses for the black solid lines in Fig. 2(b). (a-b) Single-parameter bifurcation diagrams with $b=0.09$ and $Q=1.6$, respectively, (c-d) The top two nontrivial Floquet multipliers along with the unstable branches 1 and 2 in Fig. 5(a), respectively, where $\left|\lambda_{1}\right| \geq\left|\lambda_{2}\right|$ holds.

Fig. 6 Single-parameter bifurcation diagrams along the black solid lines in Fig. 2(c). (a-b) the case of $\tau=2$ with $b=0.1$ and $Q=1.6$, respectively.

Fig. 7 Bifurcation analyses for the black solid lines in Fig. 2(d). (a-b) Single-parameter bifurcation diagrams with $b=0.06$ and $Q=1.56$, respectively. The inserted windows represent the phase diagrams, where the $X$-axis denotes the state variable $v$ and the $Y$-axis denotes the state variable $u$. The red circles in the phase diagrams indicate the mapping point in Poincaré section, (c) Enlargement view of region I in Fig. 7(a), where the blue dots are derived from the direct numerical integration and the red circles are derived from the time domain collocation method, (d) The top two nontrivial Floquet multipliers along with the red circles in Fig. 7(c), (e-f) Variation of the harmonic components in the analytical solutions of the red 
periodic responses in Fig. 7(c) with respect to parameter $Q$. The symbol $c_{i, k}, i=1,2$ represent the harmonic amplitude for the state variables $u$ and $v$, and $k$ denotes the $k$ th harmonic order.

Fig. 8 Bifurcation analyses for the black solid lines in Fig. 2(e). (a-b) Single-parameter bifurcation diagrams with $b=0.15$ and $Q=1.3$, respectively, (c) The top two nontrivial Floquet multipliers for the $N 3$ type periodic response in region II of Fig. 8(a), (d-e) The top two nontrivial Floquet multipliers for the N1 and N3 type periodic responses in region III of Fig. 8(a), respectively.

Fig. 9 Bifurcation analyses for the black solid lines in Fig. 2(f). (a-b) Single-parameter bifurcation diagrams with $b=0.2$ and $Q=1.67$, respectively, (c) Bifurcation results in region IV of Fig. 9(a), which are obtained from the multiple scales method and the details can refer to Ref. [15], (d) Bifurcation results obtained from the time domain collocation method.

Fig. 10 Basin of attraction of coexisting attractors for the case in Fig. 4(a). The black ellipses are marked in Figs. 10(b-c) for the purpose of presenting a clearer view in the change of basin area of attraction. (a) $(b, Q)=(0.2,1.12)$, $(b, Q)=(0.2,1.14),(\mathrm{c}) \quad(b, Q)=(0.2,1.15),(\mathrm{d}) \quad(b, Q)=(0.2,1.17)$.

Fig. 11 Basin of attraction of coexisting attractors for the case of Fig. 8(a). (a)

$$
\begin{aligned}
& (b, Q)=(0.15,1.67), \quad \text { (b) } \quad(b, Q)=(0.15,1.69), \quad(\text { c) } \quad(b, Q)=(0.15,1.72), \\
& (b, Q)=(0.2,1.74) .
\end{aligned}
$$

\section{Figures}




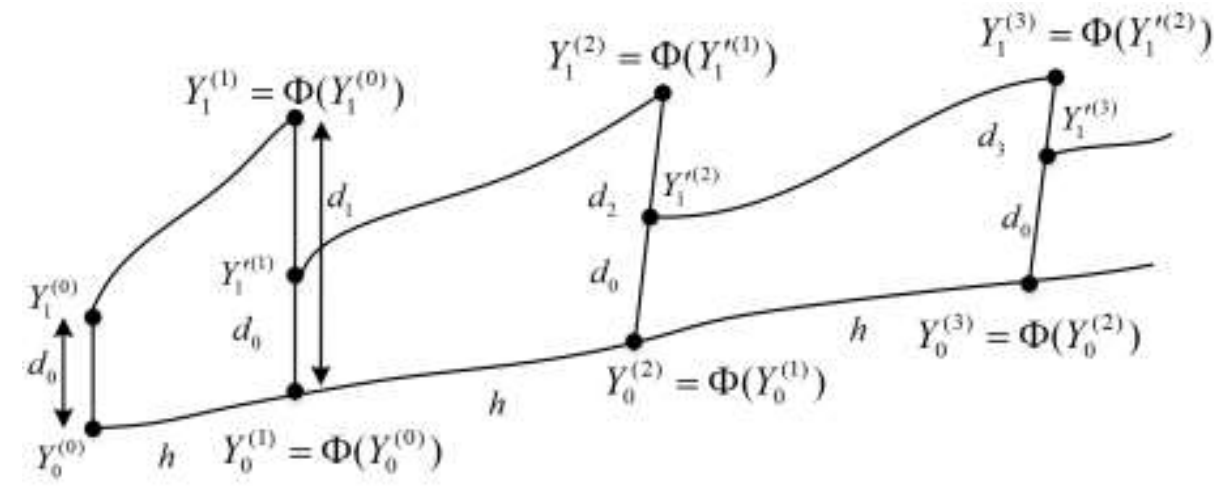

Fig. 1

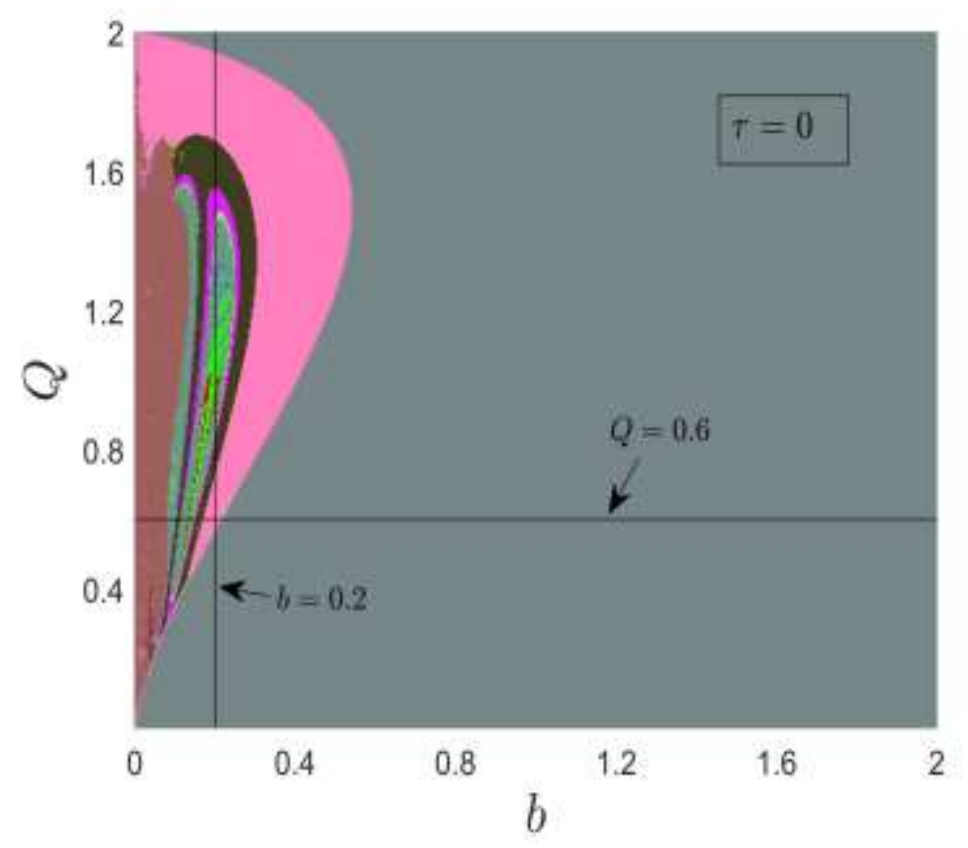

Fig. 2(a) 


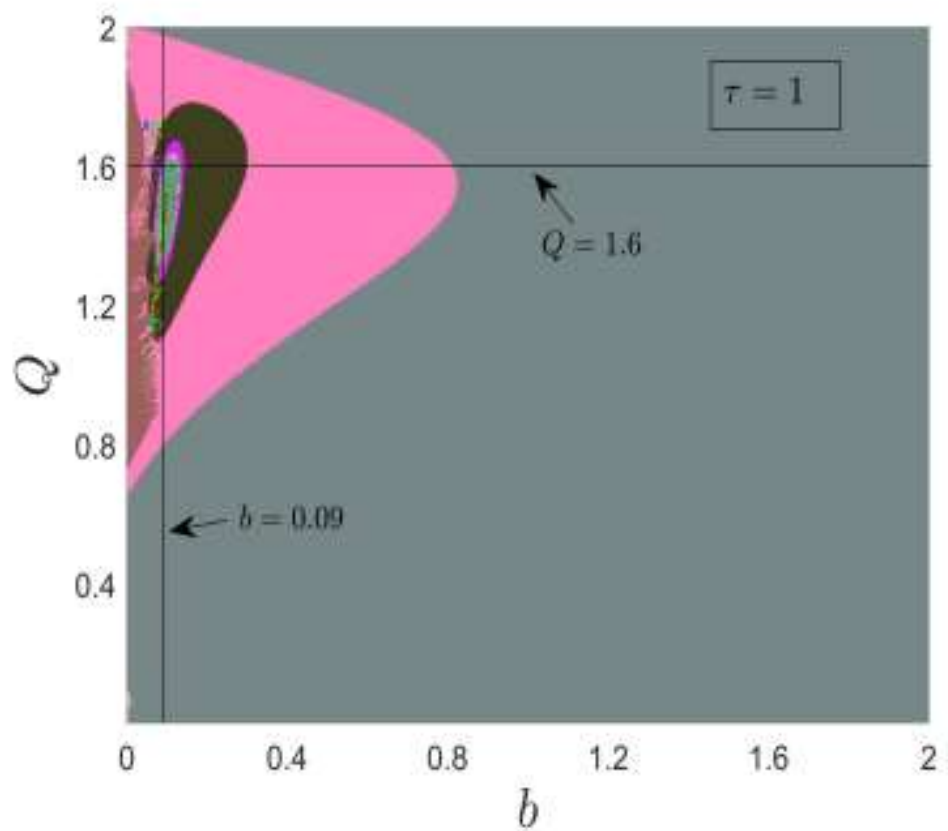

Fig. 2(b)

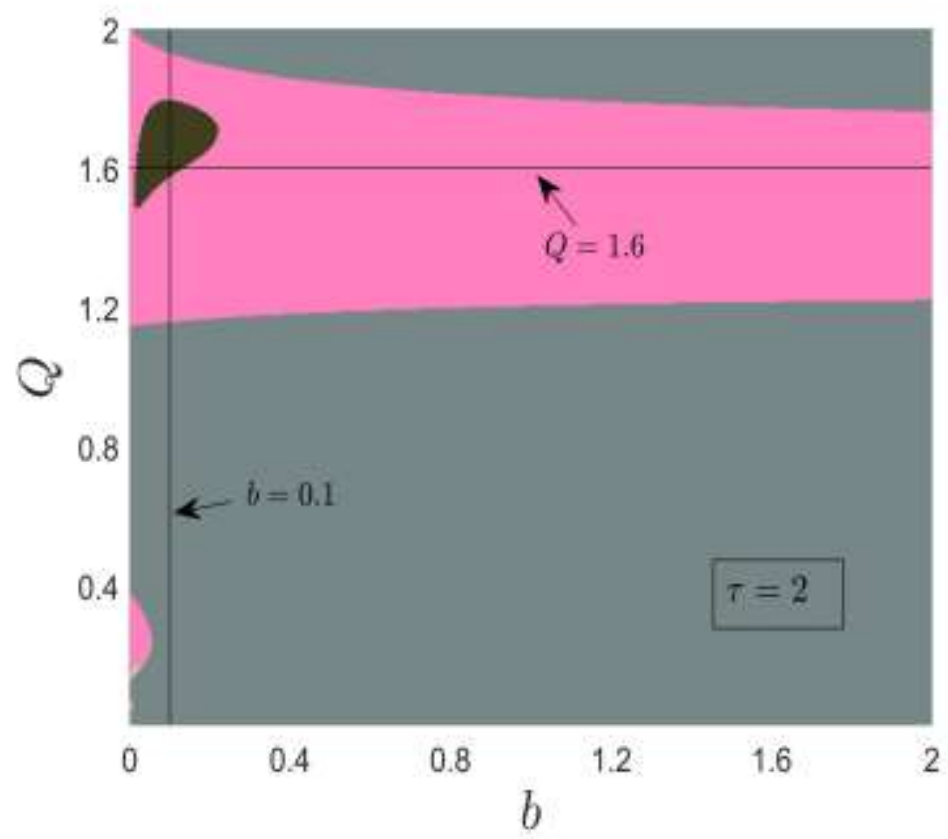

Fig. 2(c) 


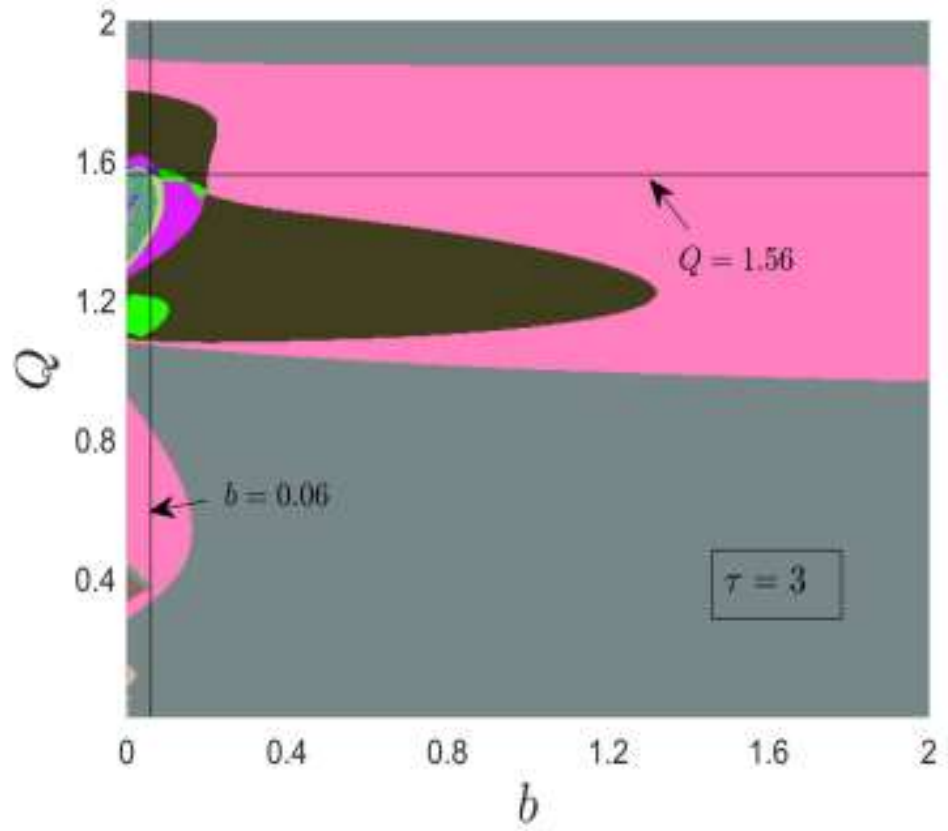

Fig. 2(d)

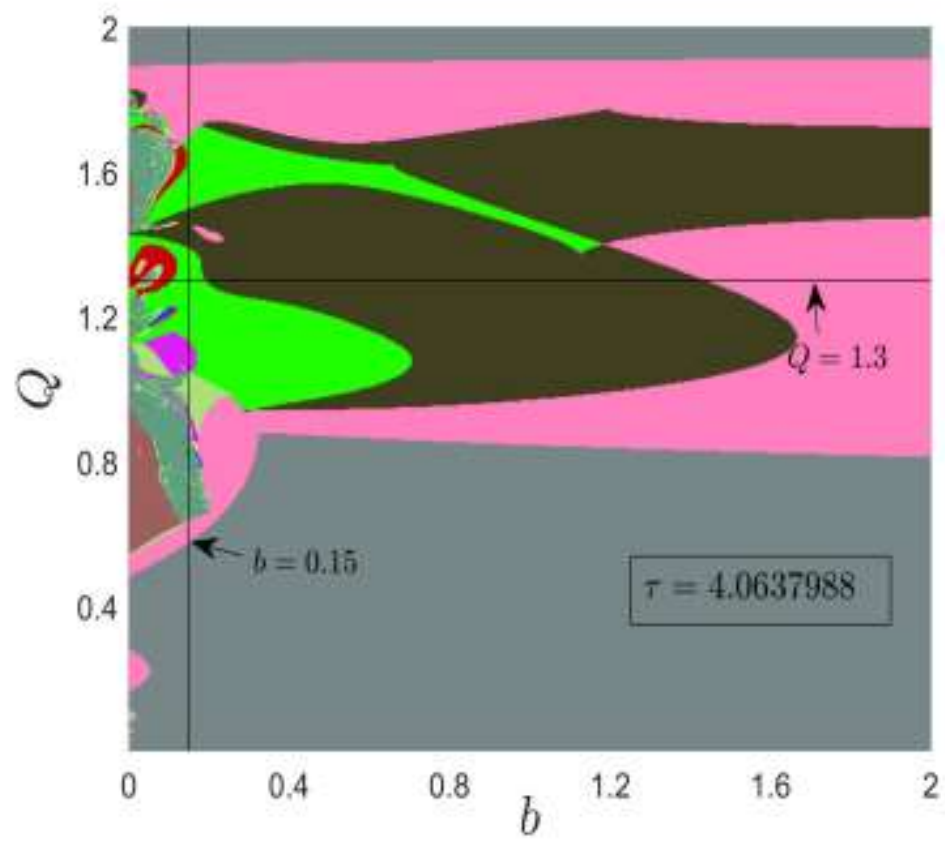

Fig. 2(e) 


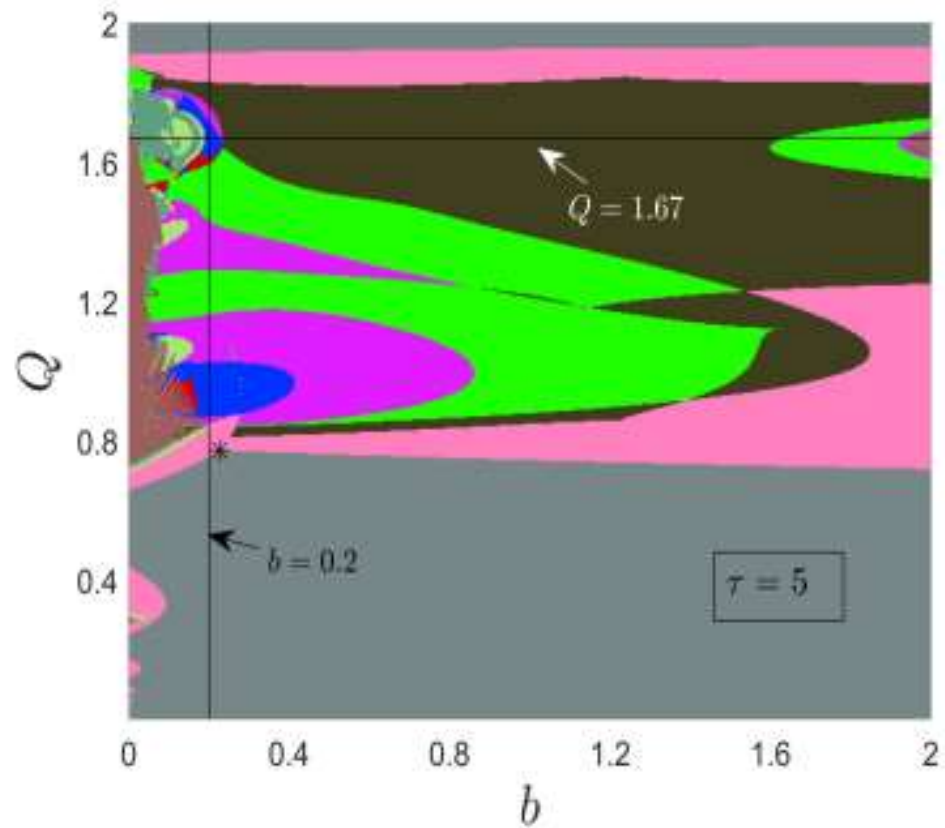

Fig. 2(f)

\begin{tabular}{|l|l|l|l|l|l|l|l|l|l|}
$D$ & $C$ & $E$ & $N 1$ & $N 2$ & $N 3$ & N4 & N5 & N6 & NQ
\end{tabular}

Fig. 2(g)

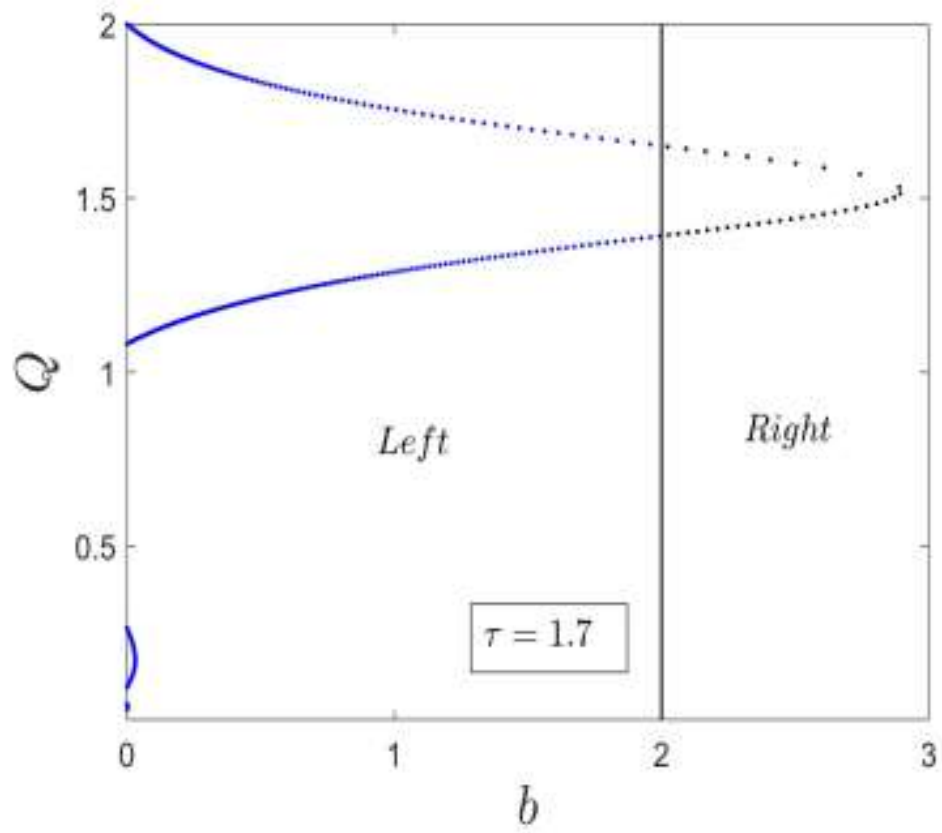


Fig. 3

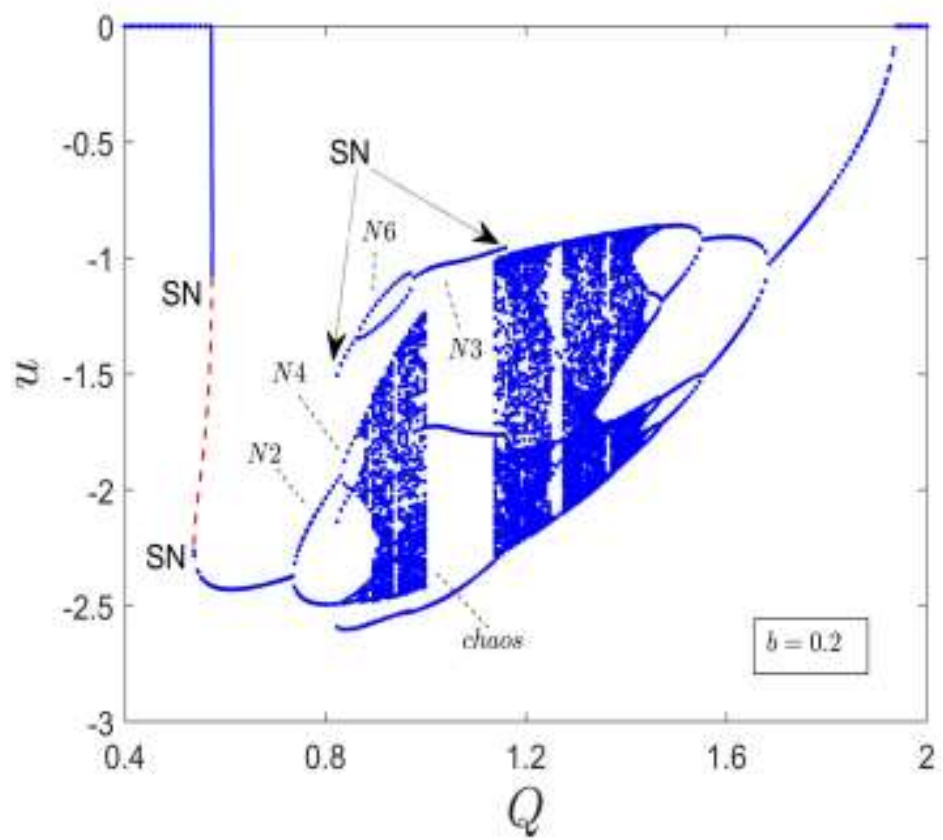

Fig. 4(a)

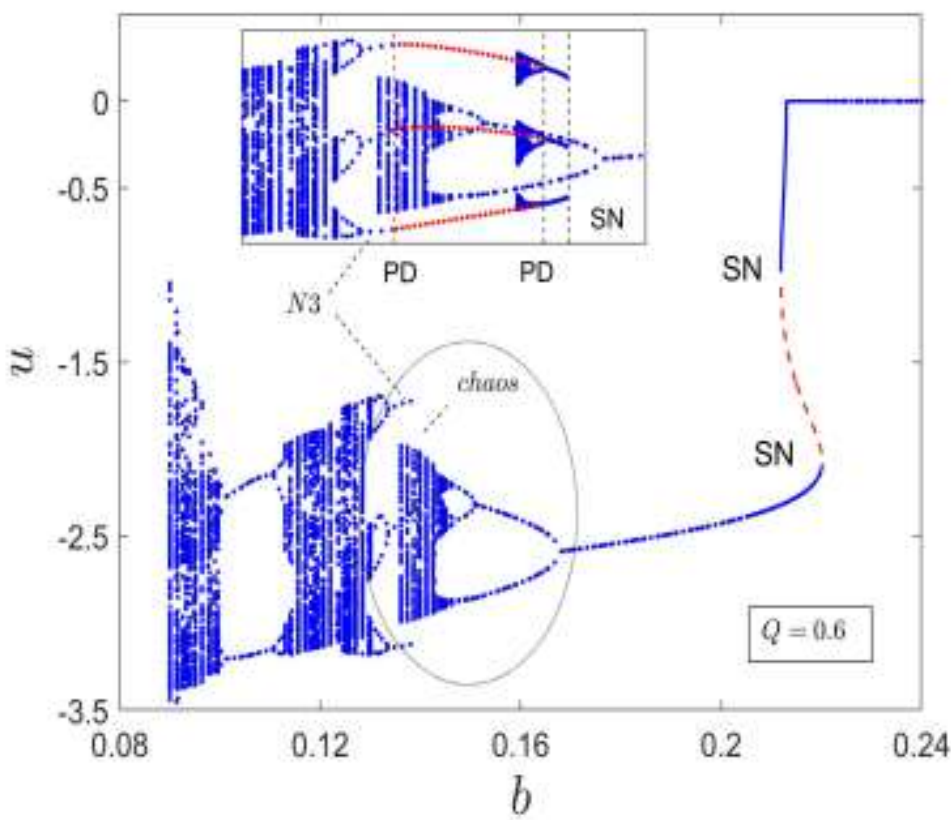

Fig. 4(b) 


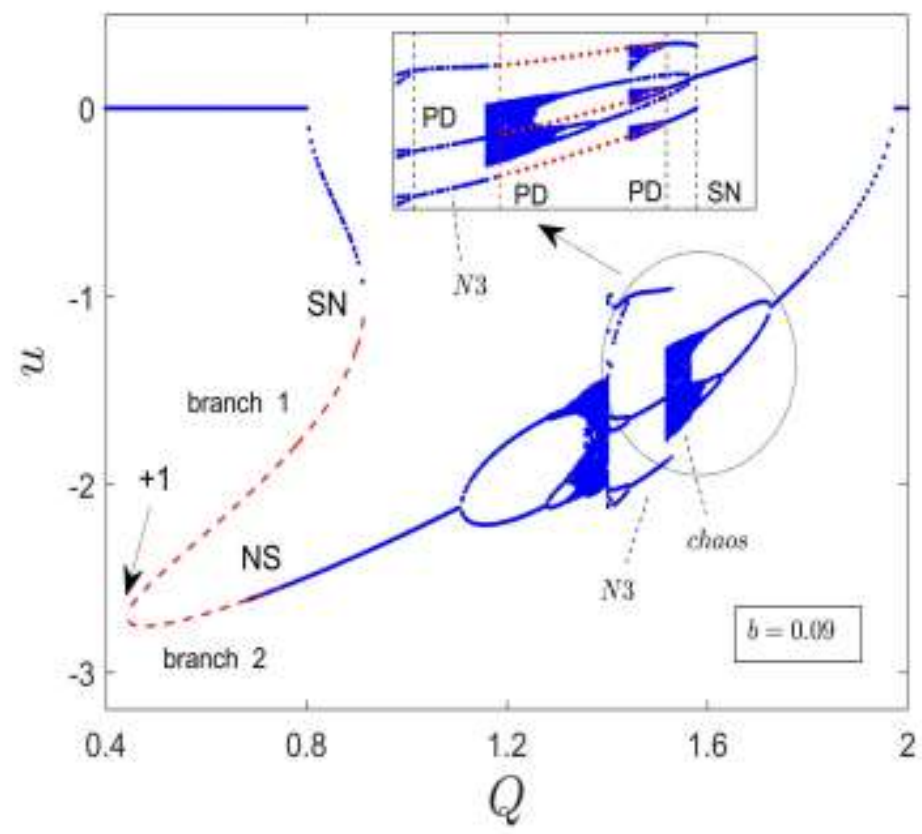

Fig. 5(a)

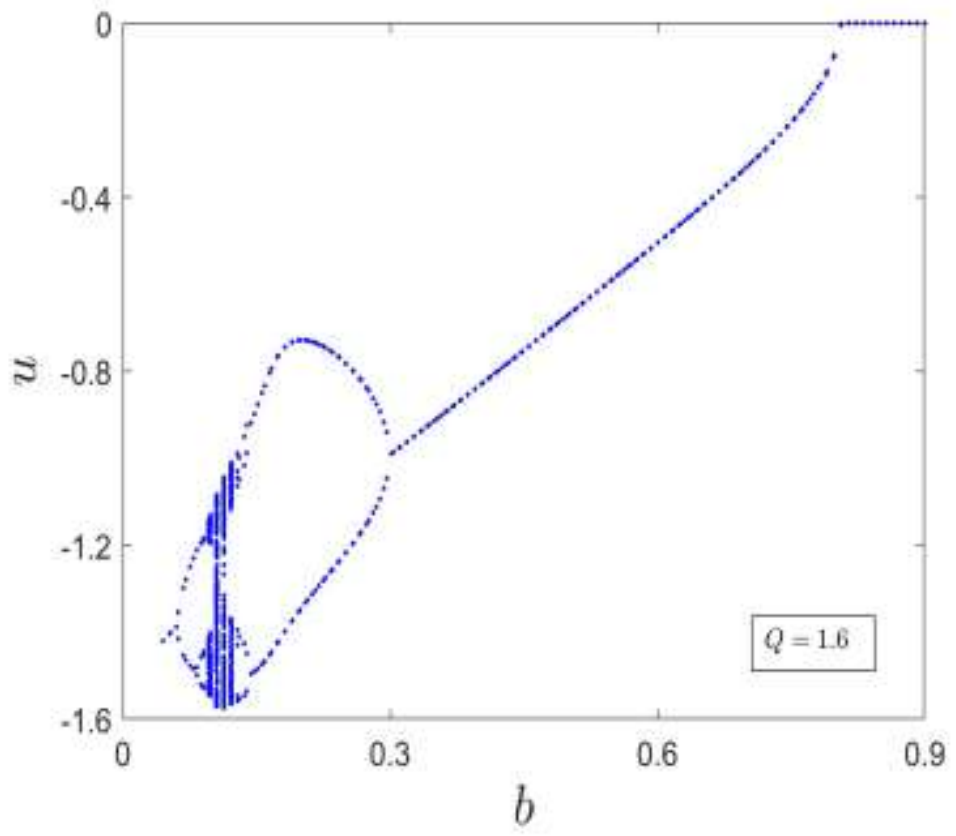

Fig. 5(b) 


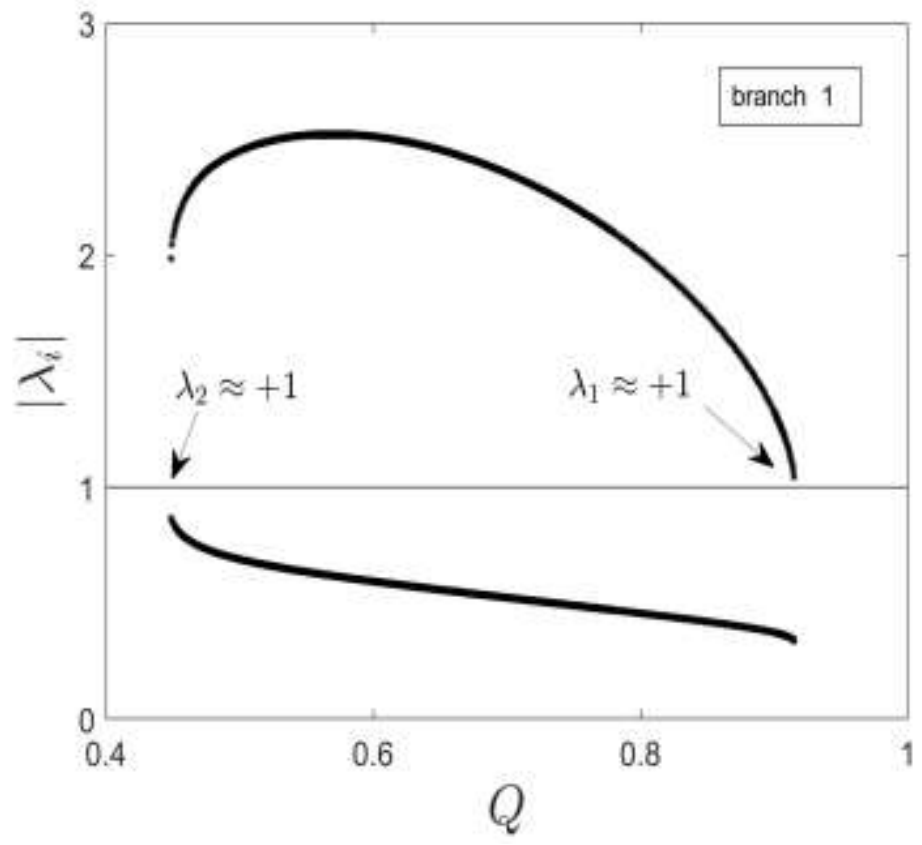

Fig. 5(c)

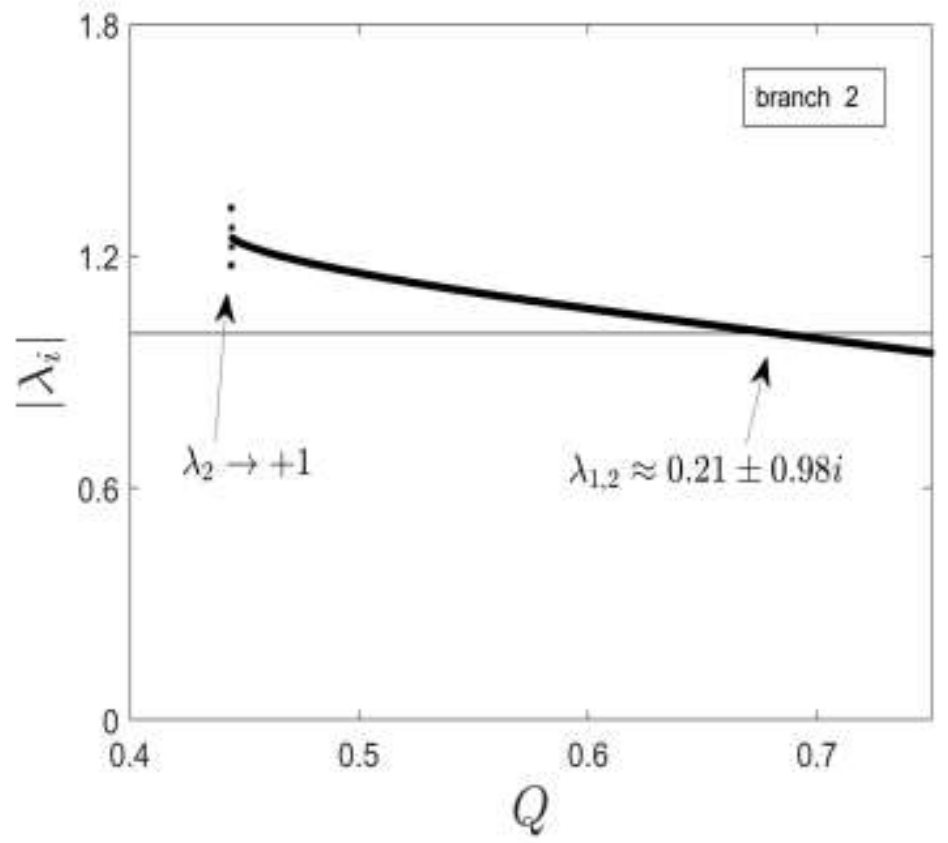

Fig. 5(d) 


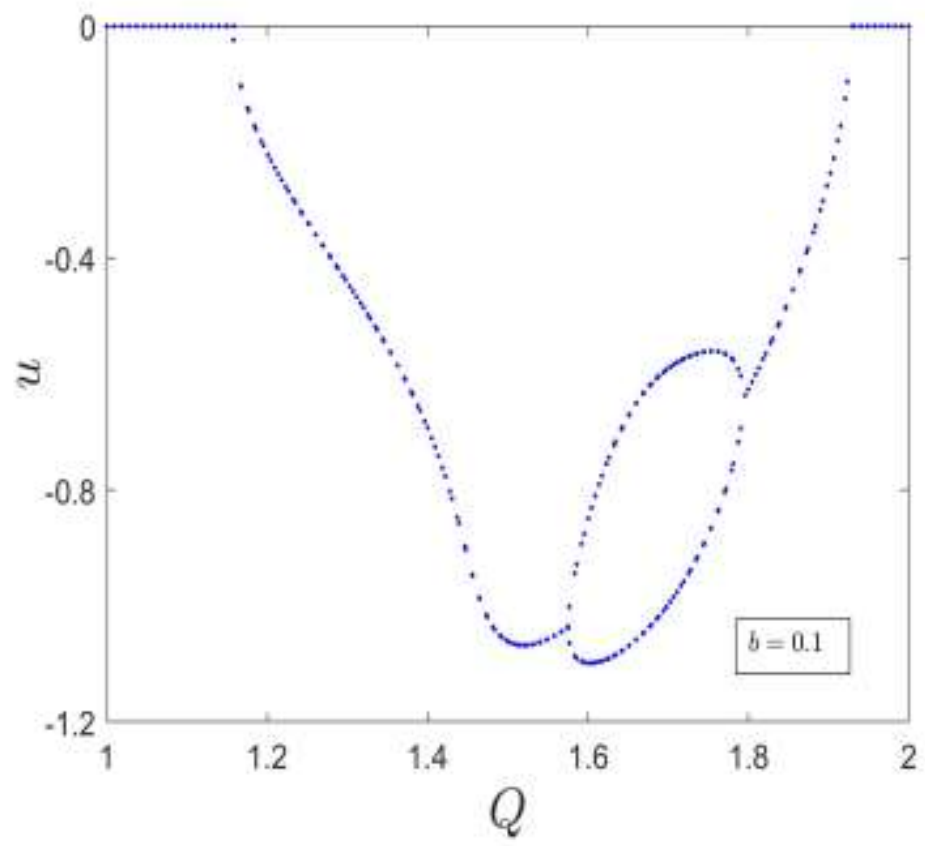

Fig. 6(a)

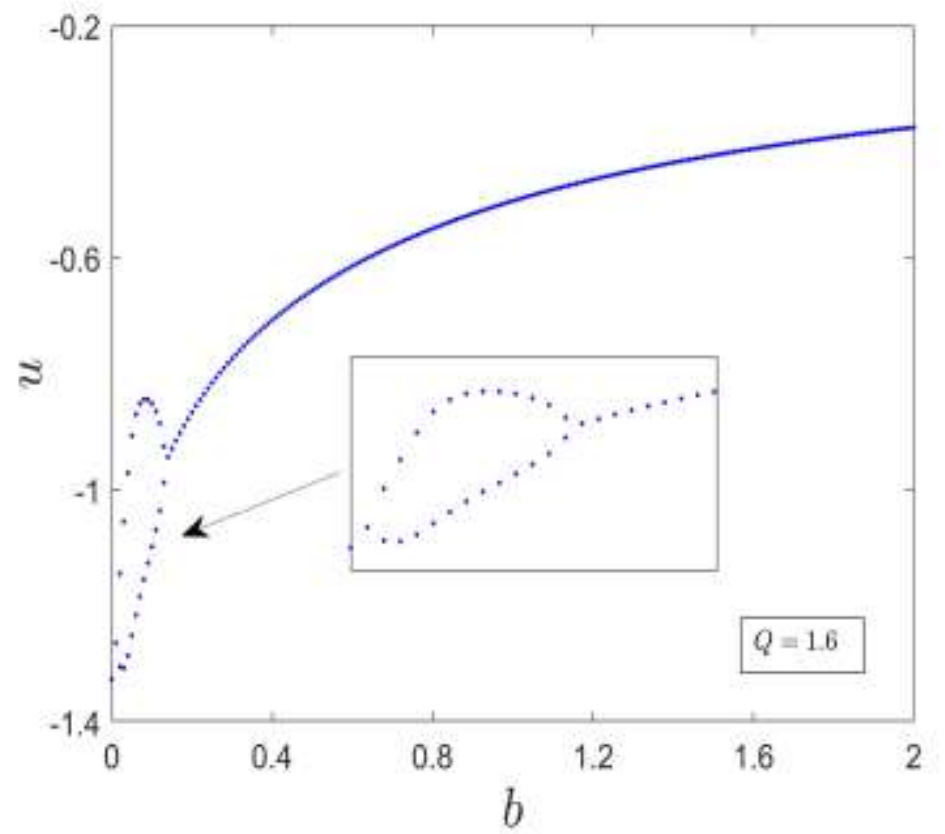

Fig. 6(b) 


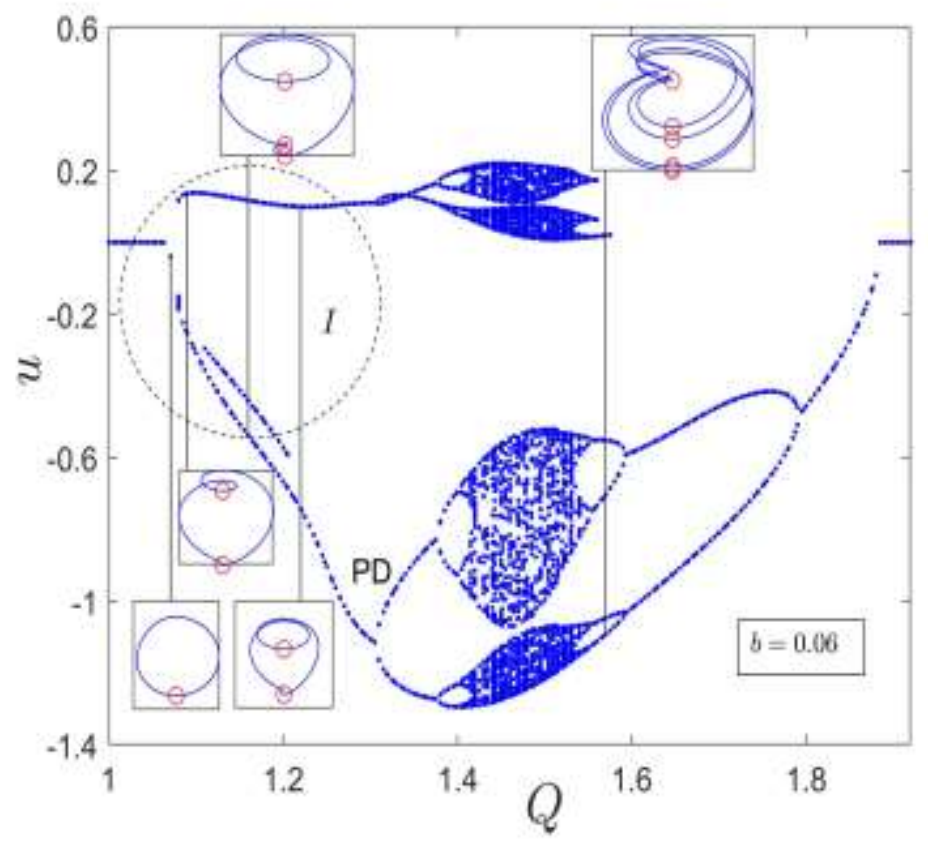

Fig. 7(a)

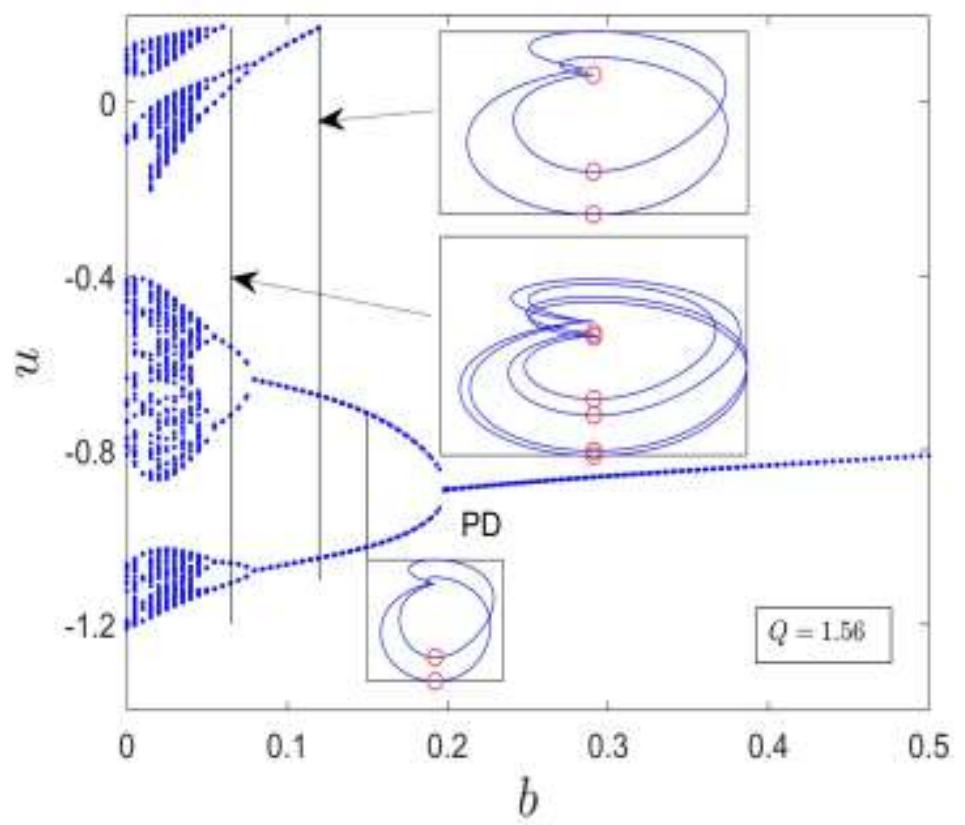

Fig. 7(b) 


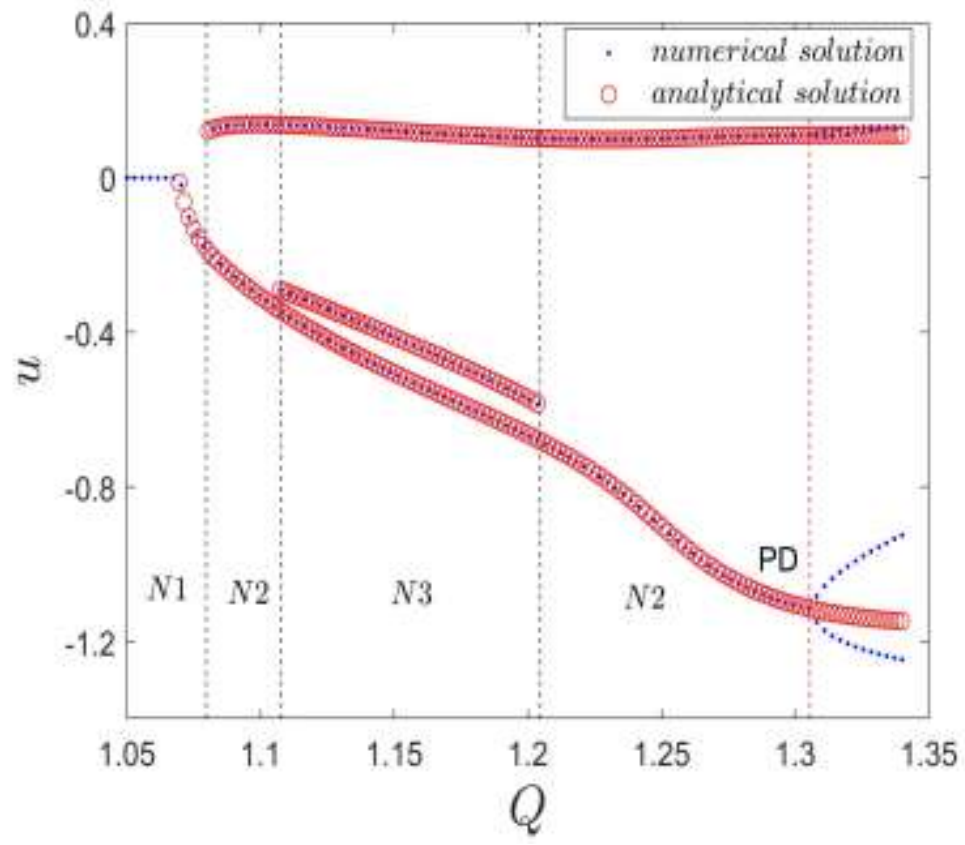

Fig. 7(c)

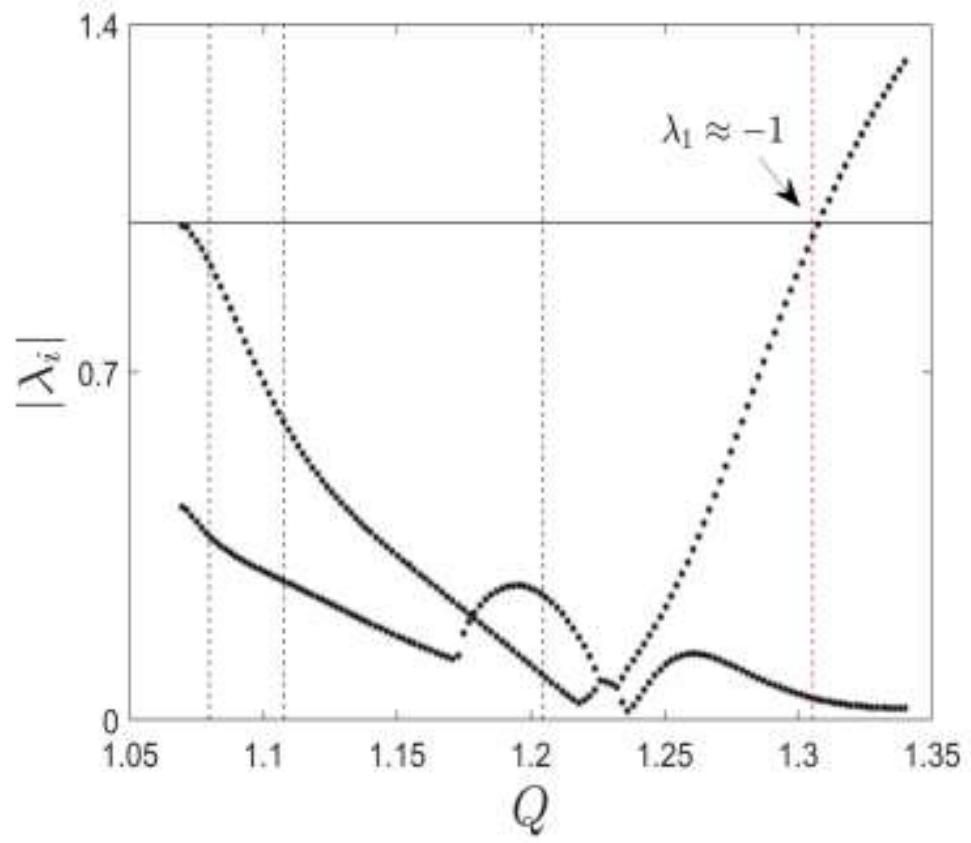

Fig. 7(d) 


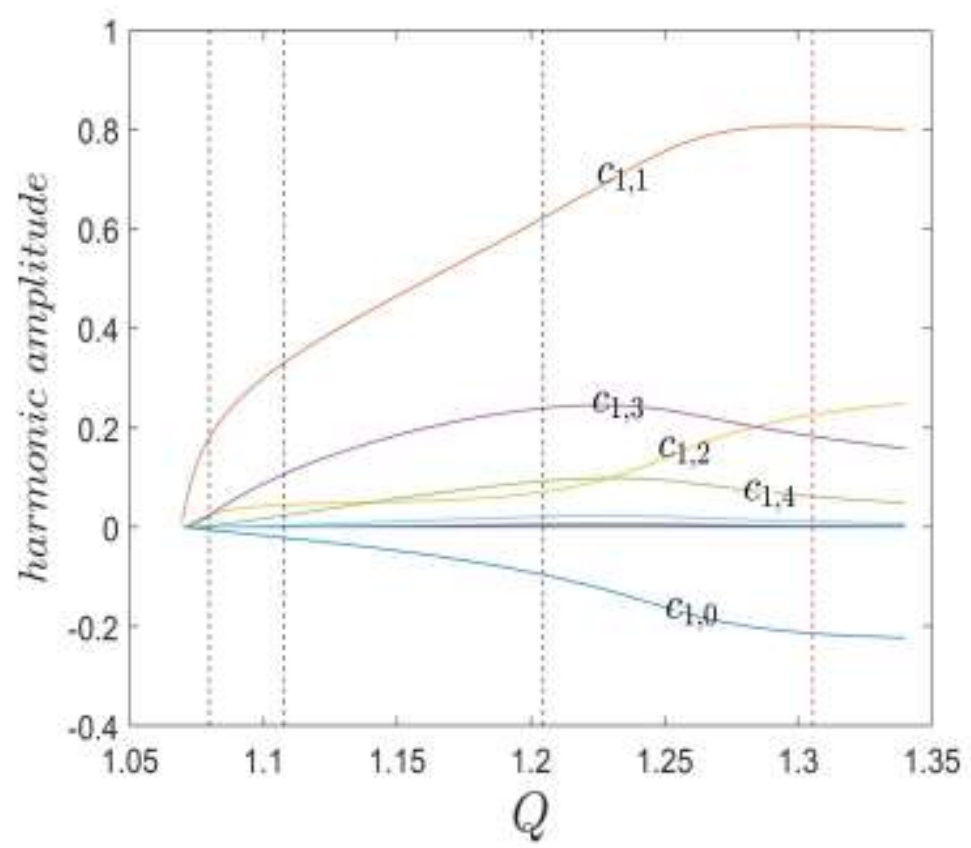

Fig. 7(e)

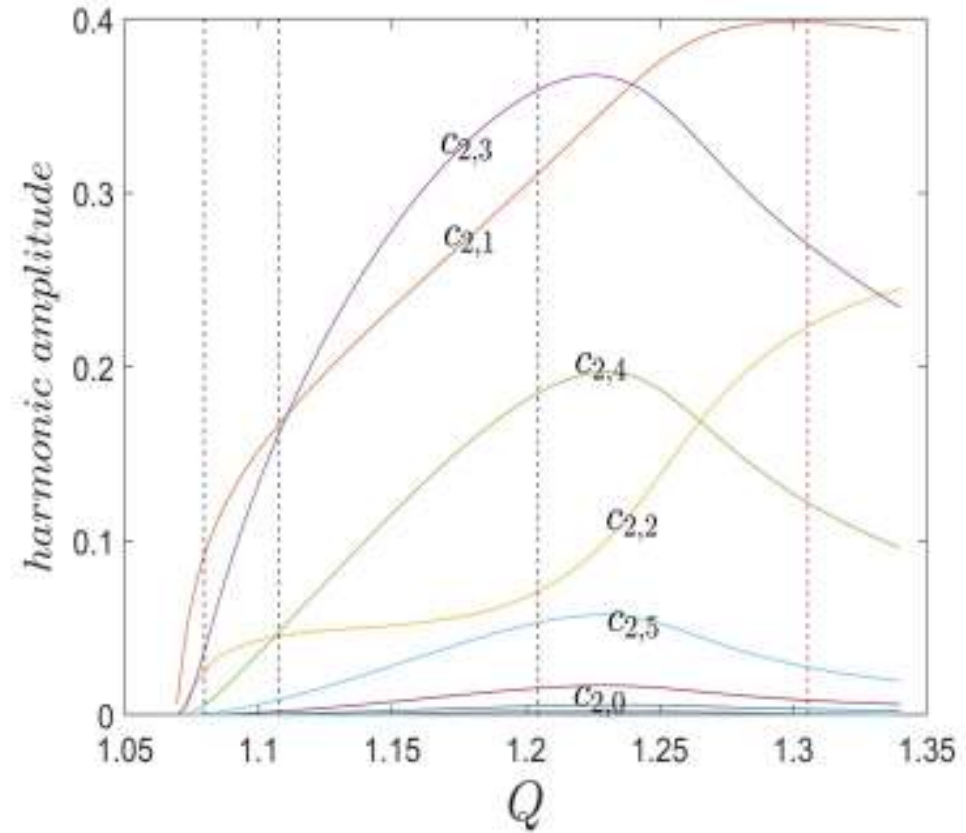

Fig. 7(f) 


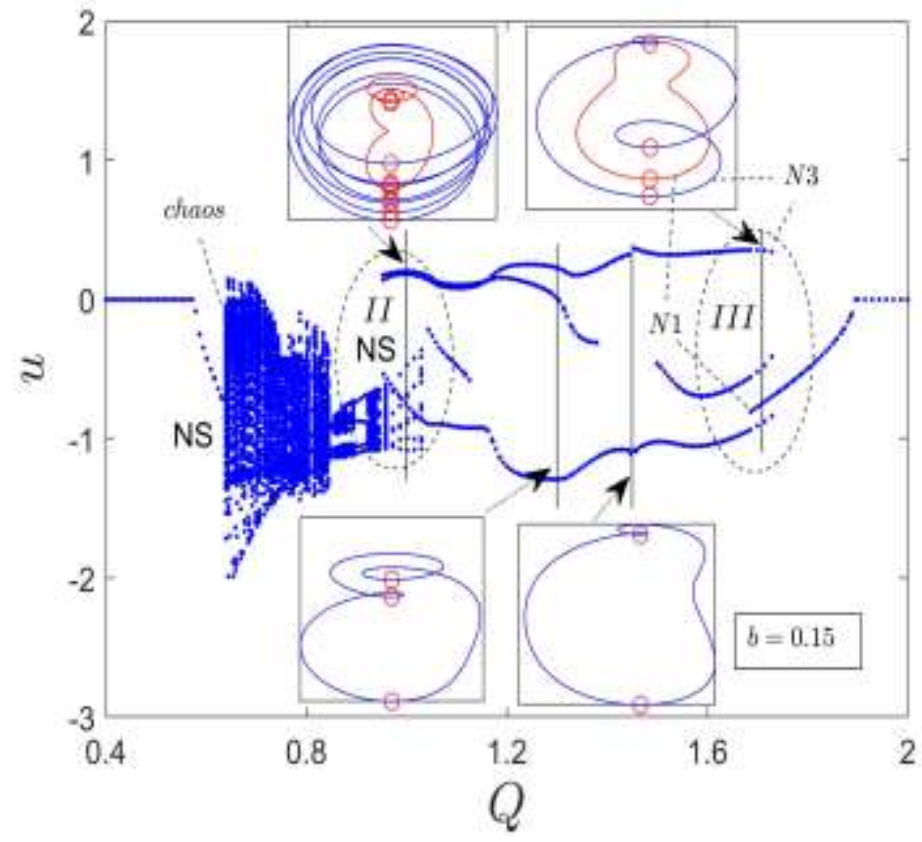

Fig. 8(a)

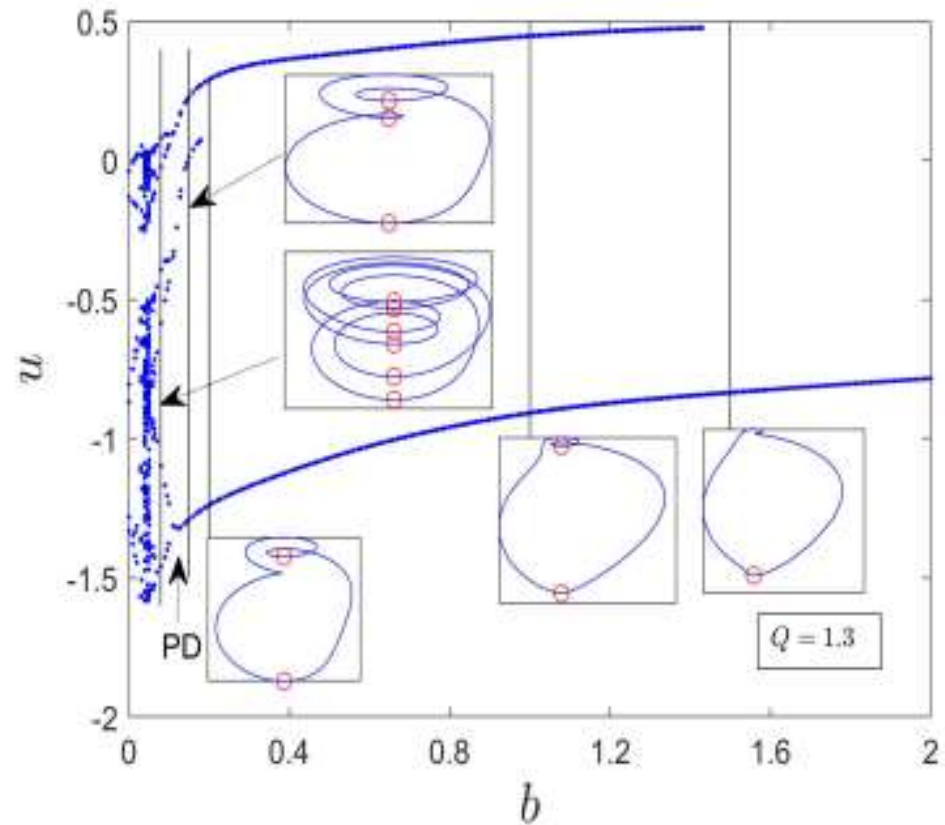

Fig. 8(b) 


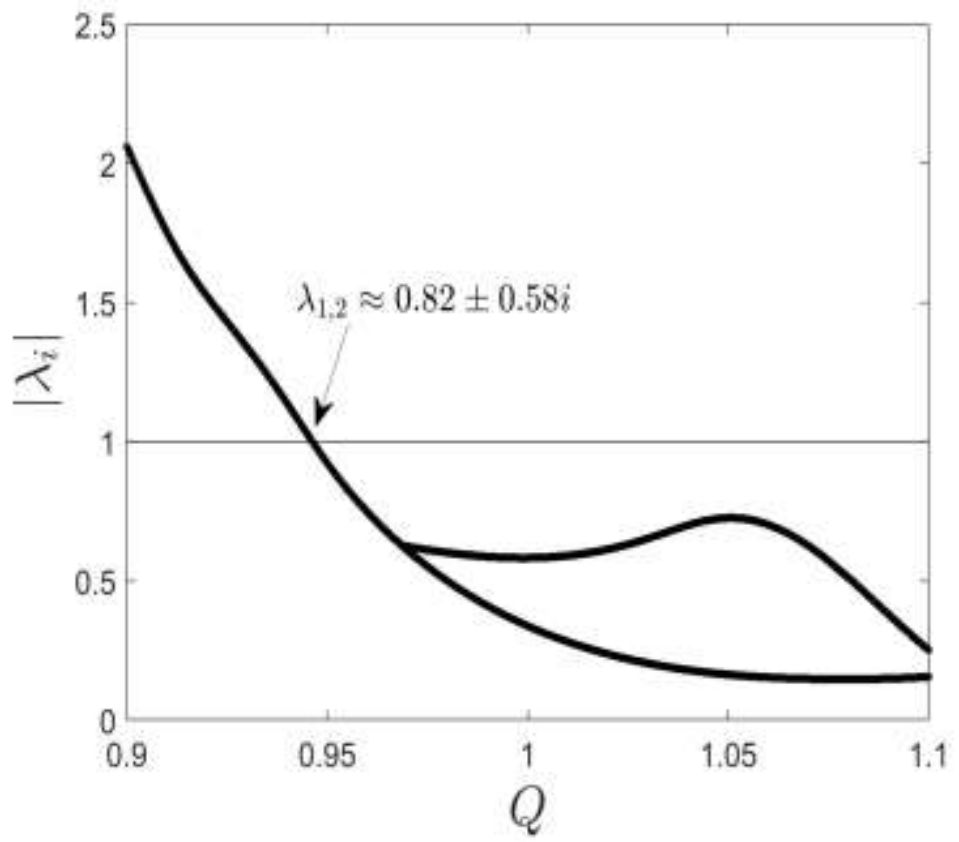

Fig. 8(c)

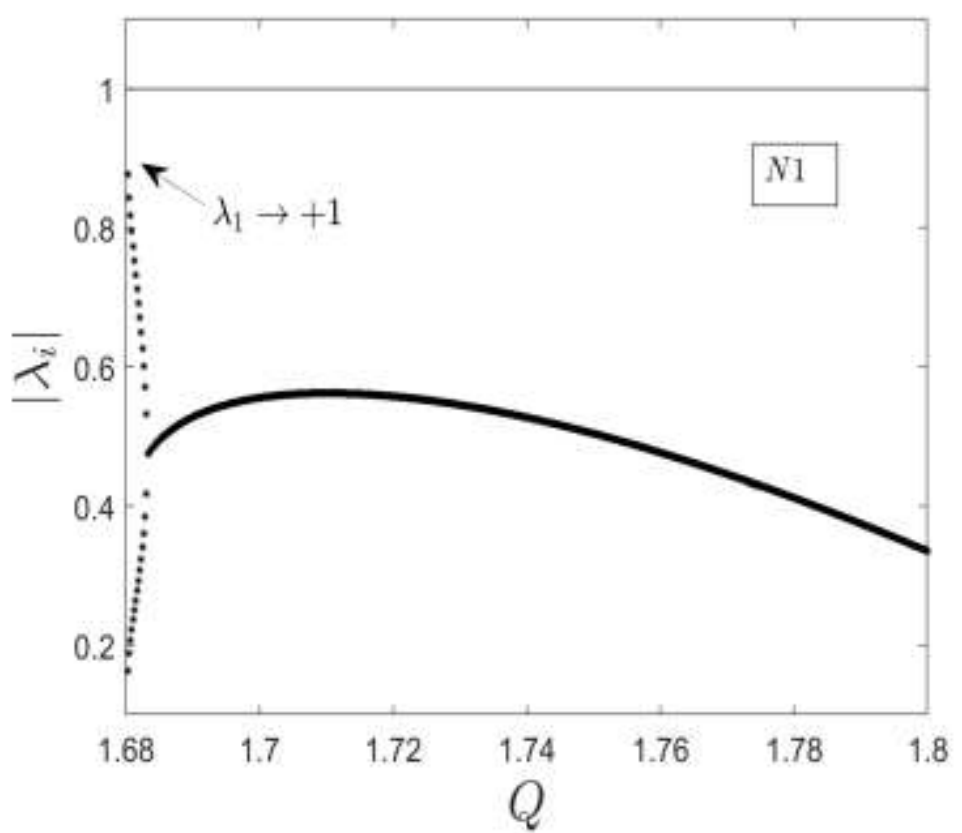

Fig. 8(d) 


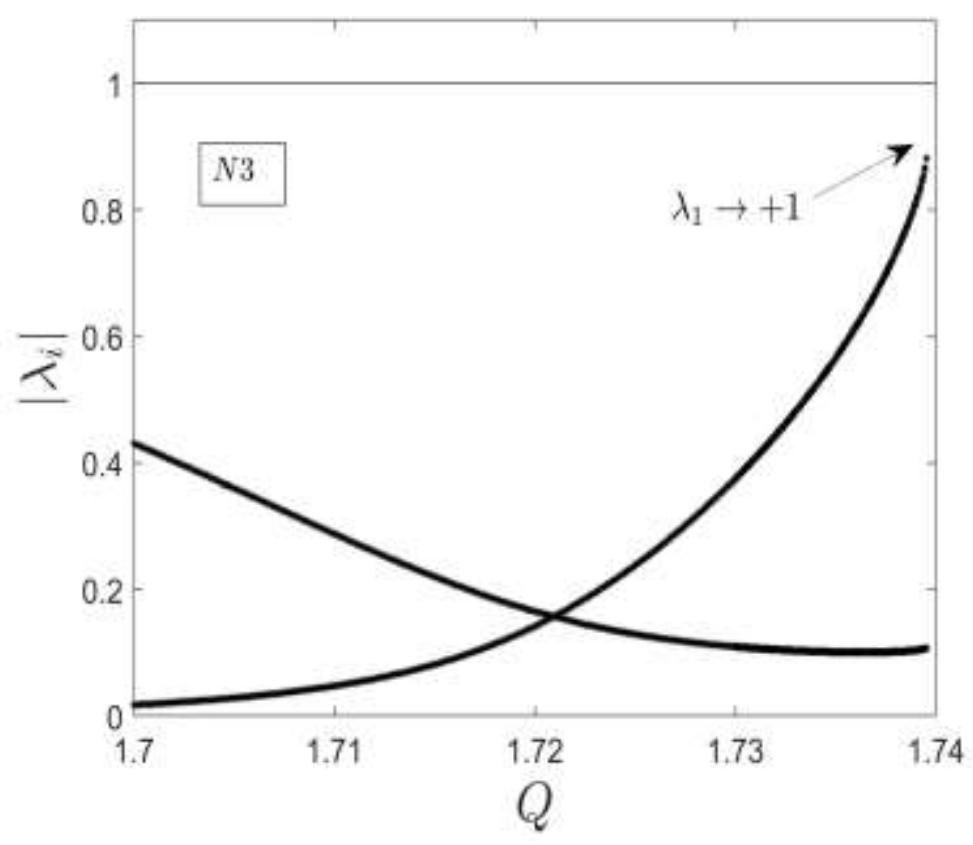

Fig. 8(e)

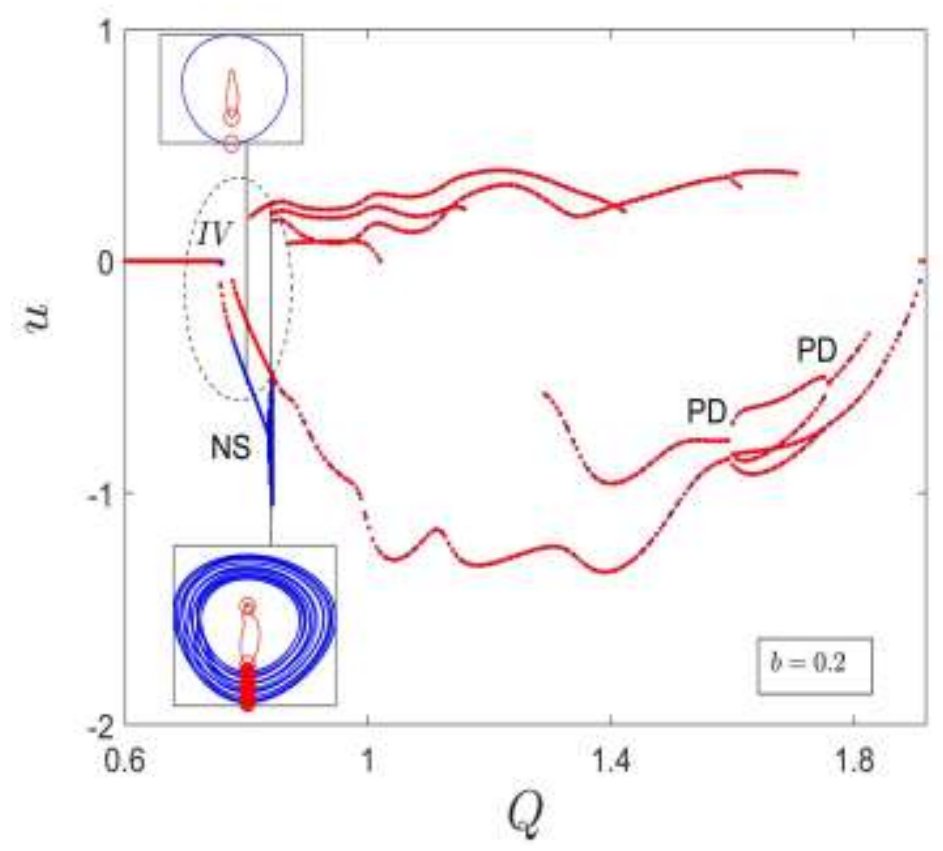

Fig. 9(a) 


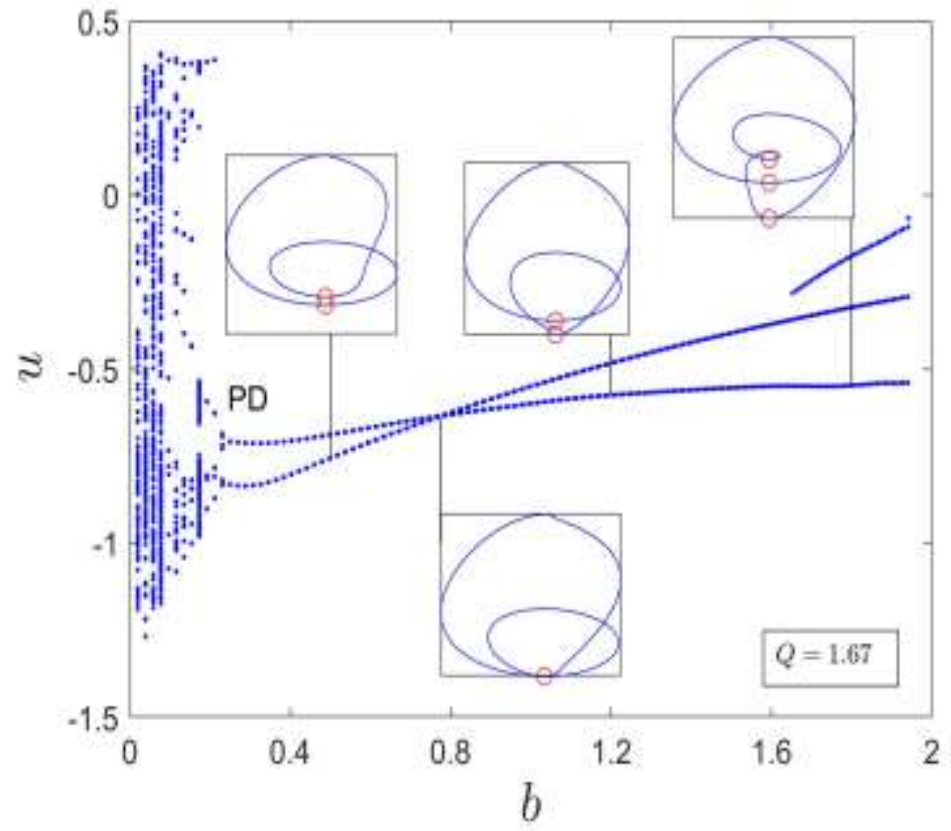

Fig. 9(b)

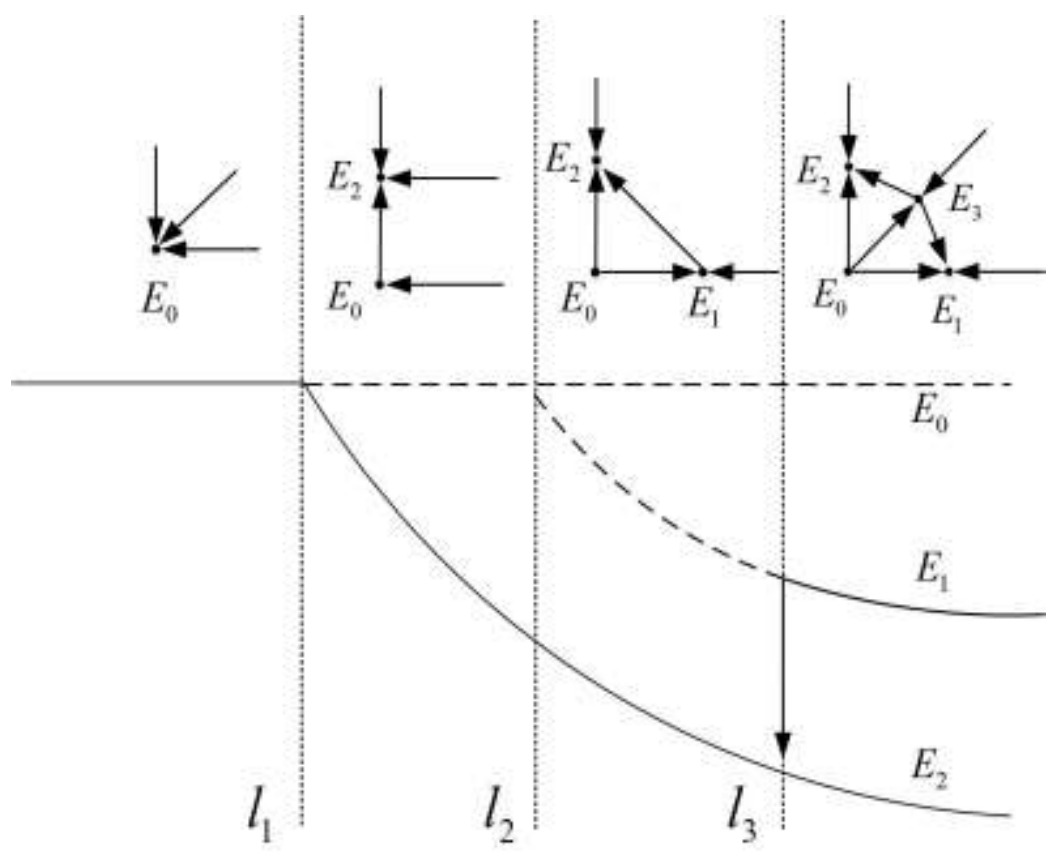

Fig. 9(c) 


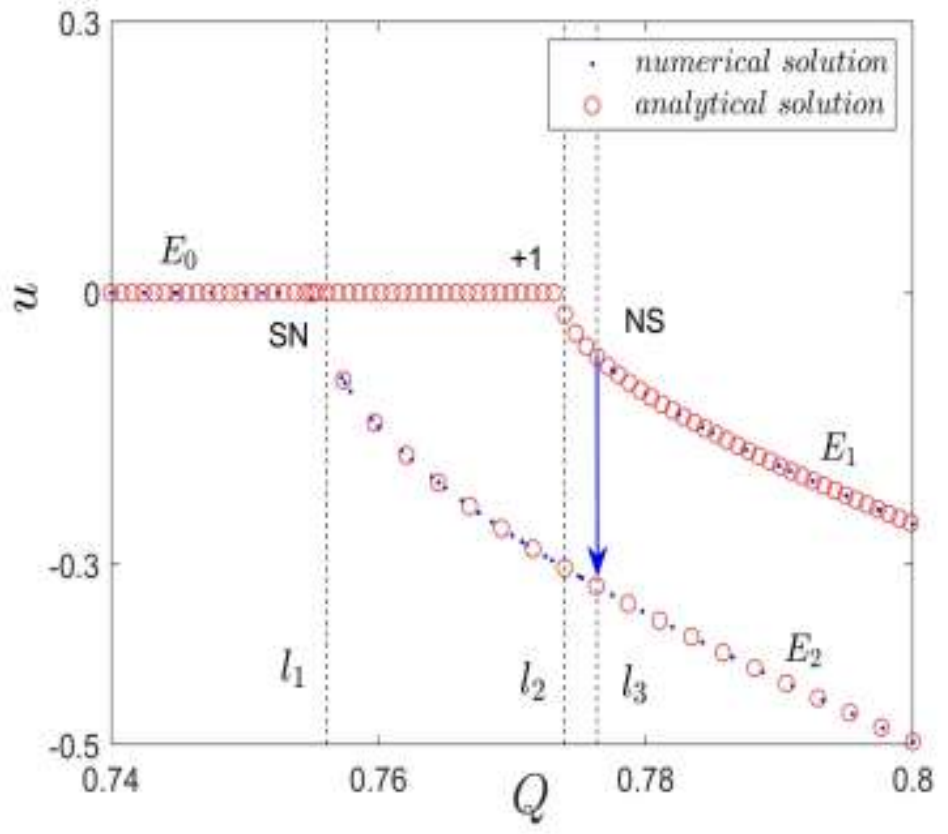

Fig. 9(d)

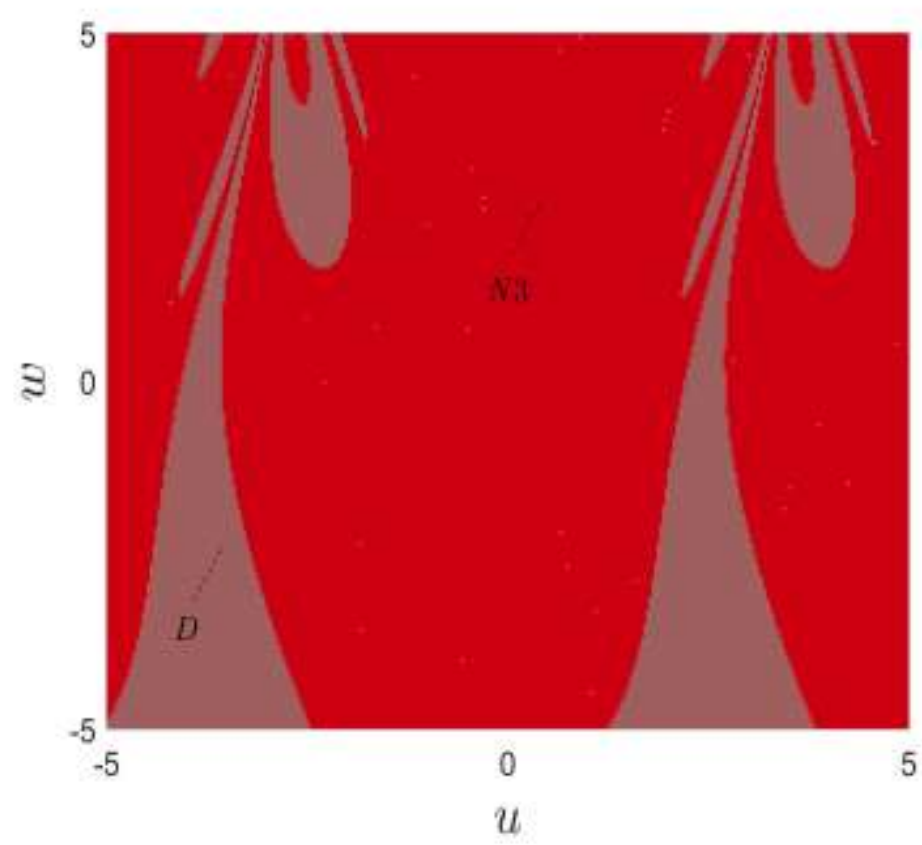

Fig. 10(a) 


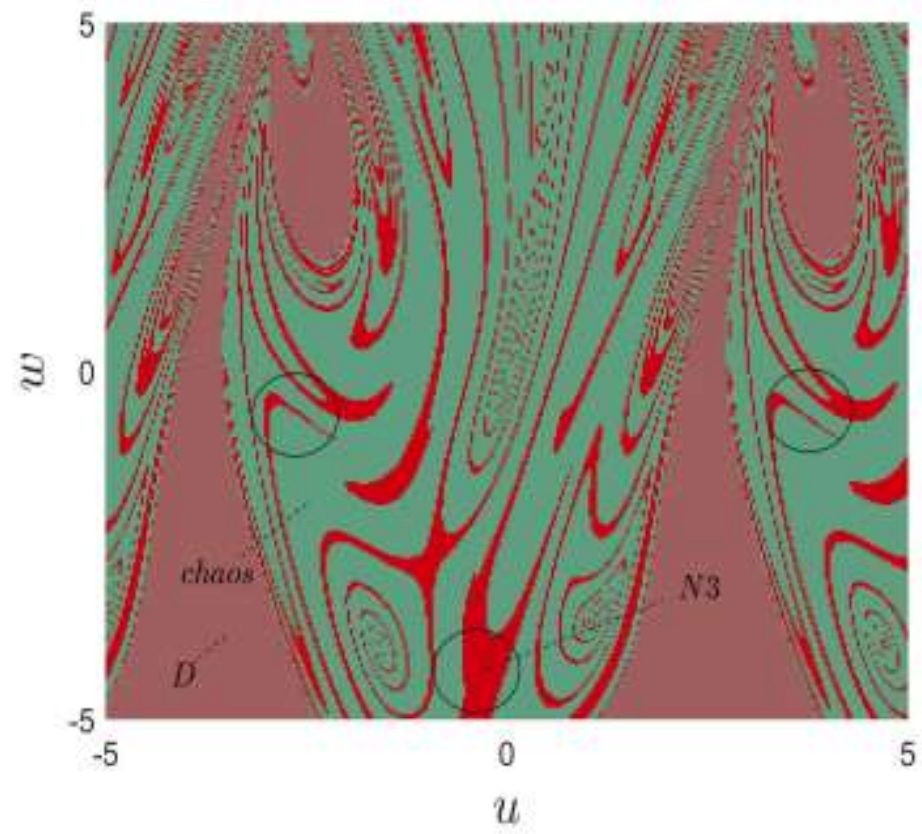

Fig. 10(b)

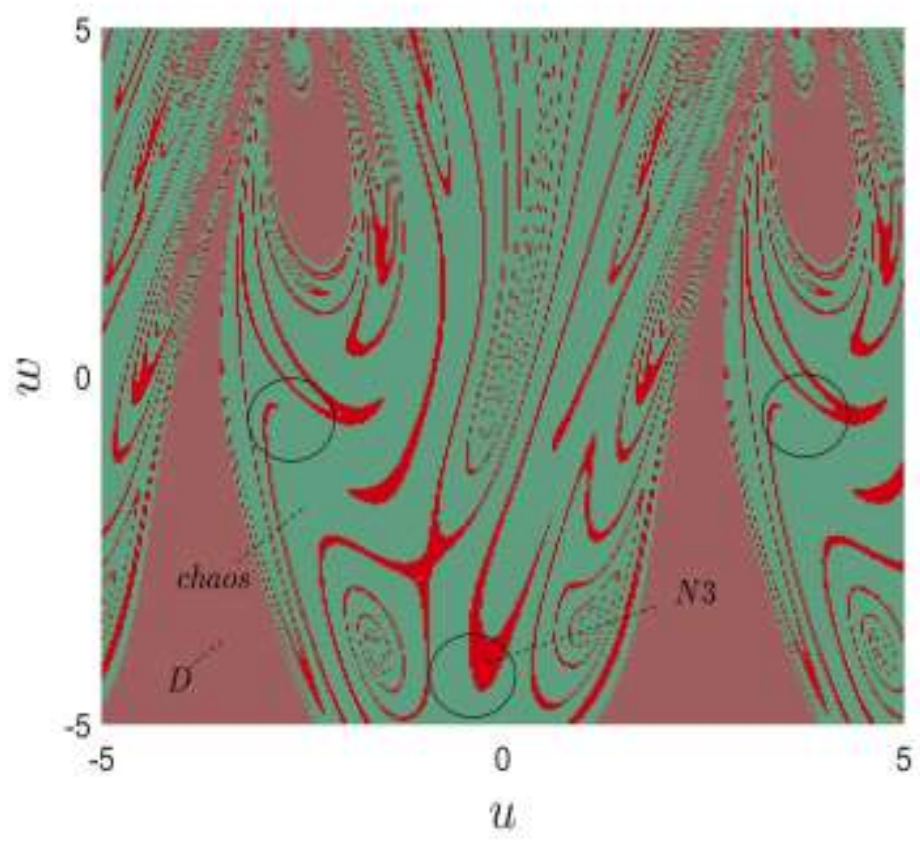

Fig. 10(c) 


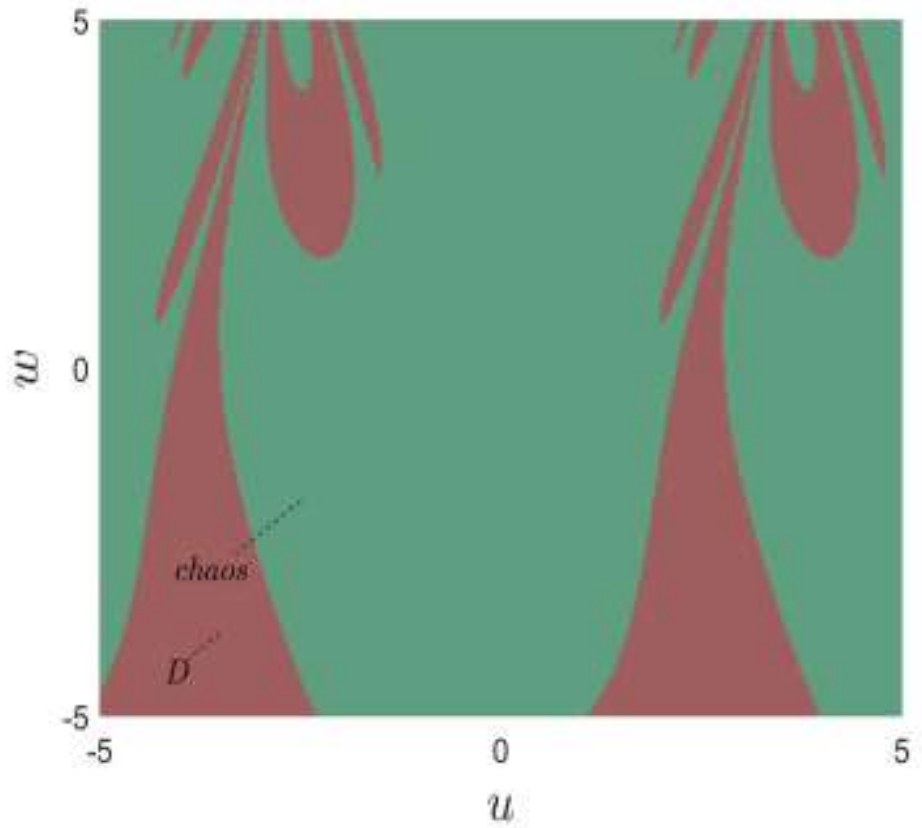

Fig. 10(d)

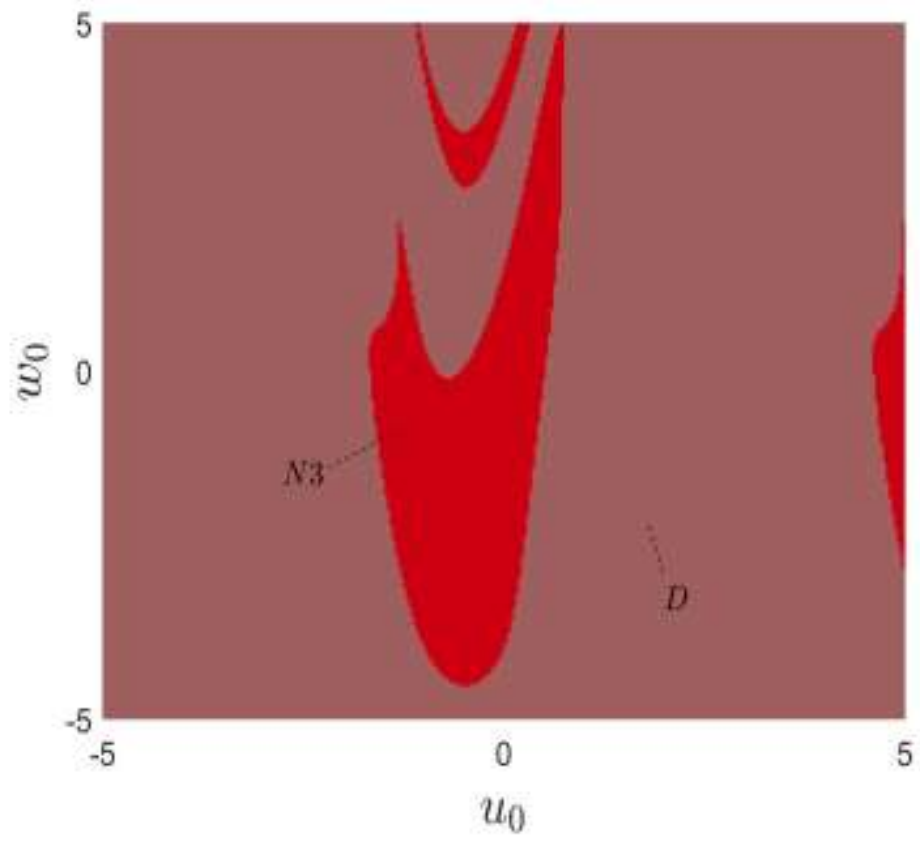

Fig. 11(a) 


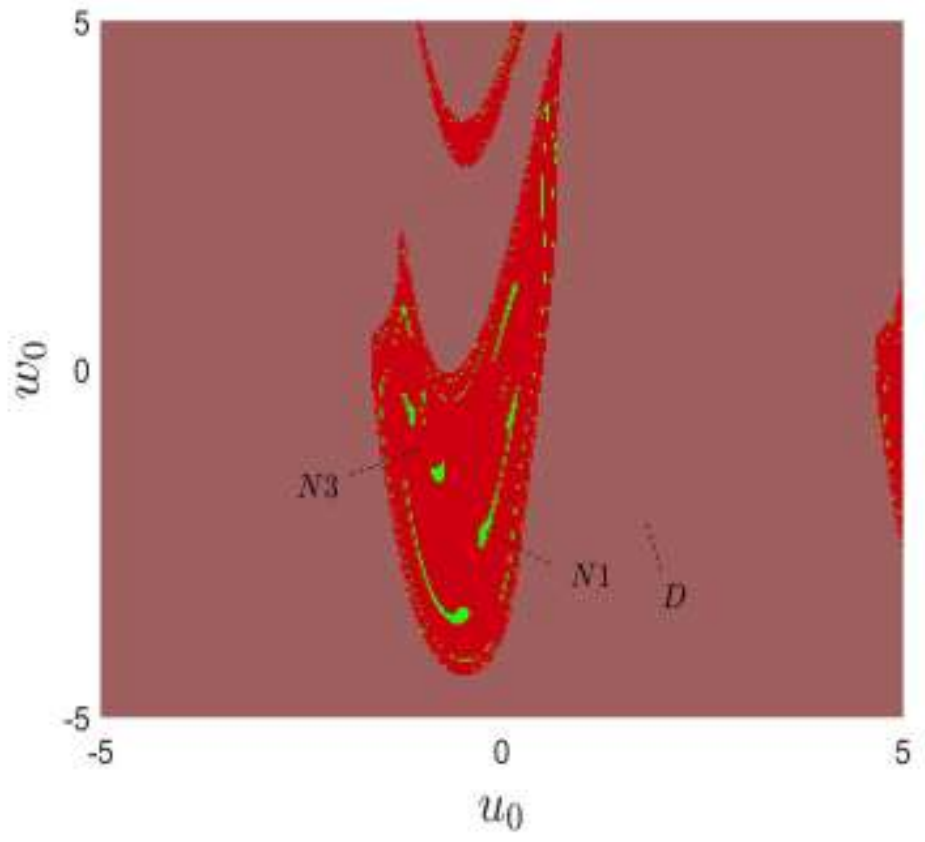

Fig. 11(b)

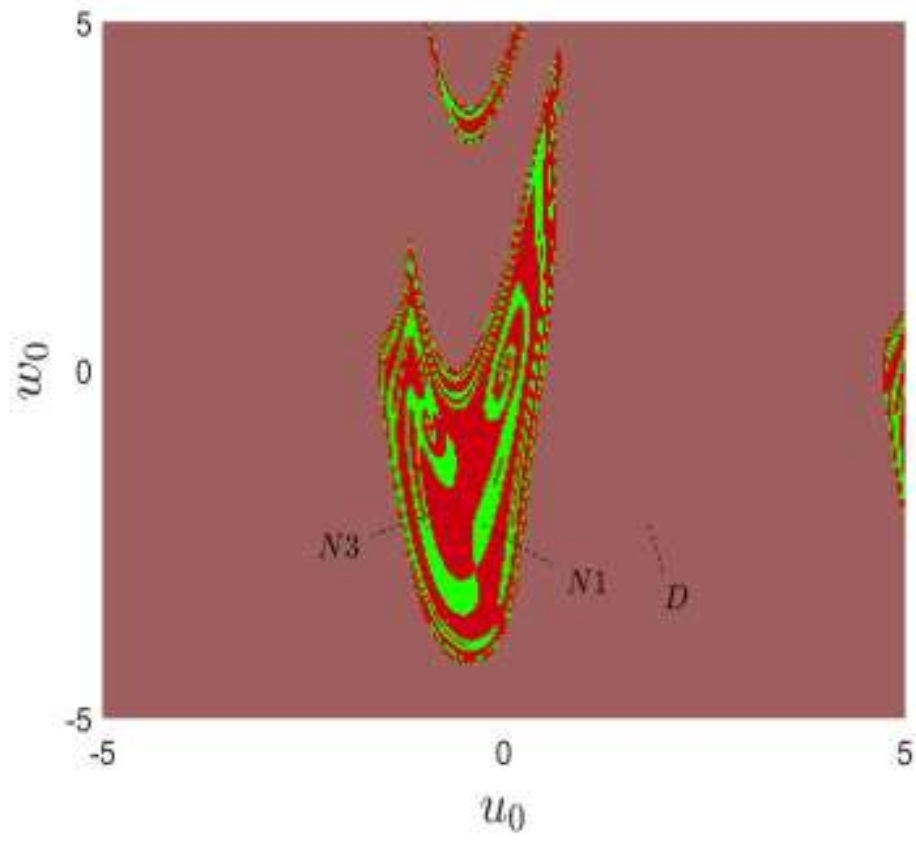

Fig. 11(c) 


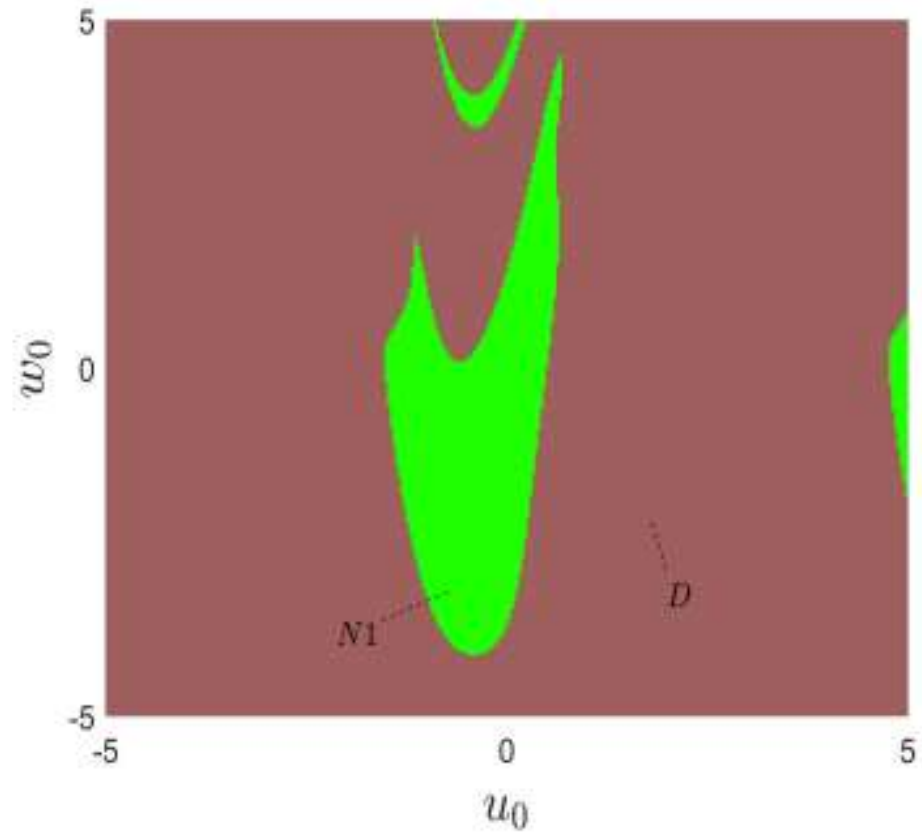

Fig. 11(d) 